\title{
The Multi-objective Steiner Pollution- Routing Problem on Congested Urban Road Networks
}

\author{
Ramin Raeesi
}

Centre for Transport and Logistics (CENTRAL), Department of Management Science, Lancaster University Management School, Lancaster LA1 4YX, UK, r.raeesi@lancaster.ac.uk

\section{Konstantinos G. Zografos*}

Centre for Transport and Logistics (CENTRAL), Department of Management Science, Lancaster University Management School, Lancaster LA1 4YX, UK,

k.zografos@lancaster.ac.uk (correspondingauthor) 


\begin{abstract}
This paper introduces the Steiner Pollution-Routing Problem (SPRP) as a realistic variant of the PRP that can take into account the real operating conditions of urban freight distribution. The SPRP is a multi-objective, time and load dependent, fleet size and mix PRP, with time windows, flexible departure times, and multi-trips on congested urban road networks, that aims at minimising three objective functions pertaining to (i) vehicle hiring cost, (ii) total amount of fuel consumed, and (iii) total makespan (duration) of the routes. The paper focuses on a key complication arising from emissions minimisation in a time and load dependent setting, corresponding to the identification of the full set of the eligible road-paths between consecutive truck visits a priori, and to tackle the issue proposes new combinatorial results leading to the development of an exact Path Elimination Procedure (PEP). A PEP-based mixed integer programming model is further developed for the SPRP and embedded within an efficient mathematical programming technique to generate the full set of the non-dominated points on the Pareto frontier of the SPRP. The proposed model considers truck instantaneous Acceleration/Deceleration (A/D) rates in the fuel consumption estimation, and to address the possible lack of such data at the planning stage, a new model for the construction of reliable synthetic spatiotemporal driving cycles from available macroscopic traffic speed data is introduced. Several analyses are conducted to demonstrate the added value of the proposed approach, exhibit the trade-off between the business and environmental objectives on the Pareto front of the SPRP, show the benefits of using multiple trips, and verify the reliability of the proposed model for the generation of driving cycles. A real road network based on the Chicago's arterial streets is also used for further experimentation with the proposed PEP algorithm.
\end{abstract}

Keywords: Time-dependent pollution-routing, Path elimination, Multiple trips, Fuel consumption, Roadway network 


\section{Introduction}

Urban Freight Distribution (UFD) is essential to the functioning of urban economies; however, it generates significant externalities such as traffic congestion and environmental pollution. European surveys indicate that the share of emissions of freight vehicles is between $20 \%$ and $30 \%$ of the total urban traffic emissions. For instance, in London in 2006 around $23 \%$ of Carbon Dioxide $\left(\mathrm{CO}_{2}\right)$ emitted by all forms of transport was due to freight vehicles (MDS Transmodal, 2012). The European Commission has therefore set a target for "essentially $\mathrm{CO}_{2}$-free city logistics in urban centres by 2030" (European Comission, 2011).

A recent survey that is aimed at assessing the target set by the European Commission (Allen, Browne, \& Piecyk, 2017) reviews freight initiatives that are expected to reduce Heavy Goods Vehicles (HGVs) kilometres and $\mathrm{CO}_{2}$ emissions in European urban areas, and ranks vehicle routing and scheduling tools among the top 10 impactful initiatives, which can help achieving around 23\% reduction in HGV vehicle kilometres by 2030. Therefore, introducing pollution related objectives into traditional Vehicle Routing Problems (VRPs) can be viewed as a major approach to combat Greenhouse Gas (GHG) emissions, and can assist decision makers to strike a balance between business and environmental objectives. This need has led to the development of a significant body of the literature related to the Emissions Minimising Vehicle Routing Problems (EMVRPs), comprising Green VRPs and the Pollution-Routing Problem (PRP) (Bektas \& Laporte, 2011). In the EMVRPs a fuel consumption estimation model, which is dependent on several vehicle and roadway network characteristics, is explicitly incorporated into the routing decision. Hence, unlike the traditional VRP that is predominantly concerned with the allocation of customers to feasible truck routes, realistic emissions minimising routing decisions on congested urban road networks must address a much more complicated decision, mainly due to the effect that the timevarying traffic conditions, the vehicle payload, and certain vehicle's physical and mechanical characteristics have on the fuel consumption level of a truck. A very first implication of this, which has not been sufficiently addressed by the previous related work in the field, is that the consideration of a priori determined single road-path for travelling between consecutive truck visits is not possible, as in practice several alternative paths can become optimal in terms of the fuel consumption between a given origin-destination pair on the underlying roadway graph depending on the departure time from the origin node, the load on the truck, and the type of the truck that is to be dispatched; none of which are known prior to realising the full route plan and schedule. Despite very recent efforts in addressing this situation (Qian \& Eglese, 2016; Ehmke, Campbell, \& Thomas, 2016; Androutsopoulos \& Zografos, 2017; Huang, Zhao, Van Woensel, \& Gross, 2017), existing approaches can only identify a limited subset of the eligible paths for the time and load dependent emissions minimisation, and it is still an open research issue to identify optimally all road-paths that must be retained.

This paper aims to close this gap by studying a new variant of the PRP, called the Steiner PRP (SPRP), directly on the original urban roadway network, and proposing new combinatorial results to develop an exact path elimination approach for the identification of the full set of the eligible road-paths (i.e. paths that might appear in a fuel consumption minimising route) in a fast pre-processing stage. It is worth mentioning that in calling the proposed variant the SPRP, we are following Cornuéjols, Fonlupt, and Naddef (1985) and Letchford, Nasiri, and Theis (2013) in calling a relevant variant of the Travelling Salesman Problem (TSP) on road networks as the Steiner TSP.

In addition to the aforementioned issue, the first step in constructing fuel-efficient truck routes involves an accurate estimation of the amount of fuel consumed at each route. Existing PRPs assign this task to a simplified average-speed version of the Comprehensive Modal Emissions Model (CMEM) formula (Barth, Scora, \& Younglove, 2004), in which all model parameters are assumed to remain constant during a truck haul, except for load and speed which might vary from one road-link to another. However, as it has been recently argued by Turkensteen (2017), CMEM is a microscopic fuel 
consumption and emission model and relies on instantaneous vehicle kinematic variables, such as second-by-second speed and Acceleration/Deceleration (A/D) rates. Lack of this information at the planning stage, especially the A/D rates, which are assumed zero over a truck haul in average-speed CMEM, can lead to an inaccurate estimation of fuel consumption and hence might lead to unreliable and misleading routing decisions. While the proposed model in this paper is developed to work directly with the instantaneous CMEM formula, to address the lack of truck A/D data we propose a simple and reliable model for the construction of synthetic driving cycles from the available macroscopic traffic speed data.

The paper also acknowledges the fact that in urban areas travel times are rather small and it is often possible that after performing short routes trucks are reloaded and used again (Olivera \& Viera, 2007). Therefore, the proposed model in the paper incorporates for the first time in the area of EMVRPs the decision regarding multiple uses of the cost and energy efficient resources during the planning horizon through the multi-trip decision-making in a multi-objective setting, where both business and environmental objectives are considered. In the SPRP three objective functions pertaining to (i) vehicle hiring cost, (ii) total amount of fuel consumed, and (iii) total makespan (duration) of the routes are considered, and to solve the problem to multi-objective optimality the paper develops a multi-phase solution framework, underpinned by the proposed exact network reduction technique, for the identification of the full set of the Non-Dominated (ND) points.

The contribution of this paper is multi-fold: (i) the SPRP is introduced as a multi-objective, time and load dependent, fleet size and mix PRP with multiple trips, time windows, and flexible departure times on congested urban road networks. In particular, the added value of the proposed model is in integrating all previously studied attributes contributing to fuel consumption, and other new important decisions such as multiple trips, into a single modelling and solution scheme, (ii) to overcome difficulties in solving the problem directly on the original roadway network, and eliminate the computational burden of the intermediate problem of finding the emissions minimising paths between consecutive visits on-the-fly, new combinatorial results are developed and used in proposing a new exact Path Elimination Procedure (PEP) that reduces the network size to a significant extent in a fast pre-processing stage by discarding all proven to be redundant paths from the network without eliminating ad-hoc ND points, (iii) to address a shortcoming of previous research in estimating fuel consumption accurately, the microscopic CMEM formula incorporating instantaneous truck kinematic variables including the time-dependent second-by-second speed and A/D rates is used in the models proposed in the paper, and (iv) a new Mixed Integer Linear Programming (MILP) model for the construction of realistic road-and-time-dependent driving cycles from macroscopic traffic speed data is proposed, to supply the model with the possible lack of the instantaneous truck A/D data at the planning stage.

The remainder of the paper is structured as follows: section 2 discusses a background on the most relevant literature. Section 3 develops the SPRP model. Section 4 elaborates on the proposed path elimination approach and the model based on it. Section 5 discusses the methodology used for the identification of ND points to the SPRP. Section 6 discusses the proposed approach for generating driving cycles. Computational experiments are presented in Section 7; and finally, section 8 concludes the paper.

\section{Previous related work}

The problem considered in this paper encompasses several attributes frequently encountered in real world urban freight distribution settings, including the time-varying road congestion, time and load dependent path selection, multiple use of the vehicles in the fleet, decisions on hiring a heterogeneous fleet of vehicles, and inclusion of both business and environmental objectives in decision making. There is research work focusing on each independent aspect of the proposed problem; however, the intention of this section is to discuss a selected review of the key studies in the general area of emissions minimising vehicle routing, and the more specific area of emissions minimisation on congested urban road 
networks. For an inclusive and up-to-date review on the state-of-the-art literature on the role of operational research in green freight transportation, the reader is referred to the recent study of Bektaş, Ehmke, Psaraftis, and Puchinger (2019). We may also refer to Ben Ticha, Absi, Feillet, and Quilliot (2018) for a review on VRPs that are studied on road networks.

In the VRPs with explicit consideration of environmental performance of the planned routes, fuel consumption is usually used as a proxy for pollutants emissions, particularly $\mathrm{CO}_{2}$ emissions which are proportional to the amount of fuel consumed. Fuel consumption is in turn dependent on many factors, and several of these factors such as vehicle type, speed, and load have already been considered in emissions minimising vehicle routing models. Some of these models only incorporate the effect of the load carried by the vehicle on the fuel consumption level of routes (Kara, Kara, \& Yetis, 2007; Xiao, Zhao, Kaku, \& Xu, 2012; Ubeda, Arcelus, \& Faulin, 2011). The main bearing on the emissions level in this category of models is due to the sequence that customers are visited which affects the payload between consecutive visits. More sophisticated models recognise the major role of the vehicle speed over each road-link in addition to the vehicle load. In the PRP (Bektas \& Laporte, 2011) and several related papers (Demir, Bektaş, \& Laporte, 2012; Kramer, Maculan, Subramanian, \& Vidal, 2015) fuel consumption is assumed a nonlinear convex function of the vehicle speed, and hence speed optimisation in a time-independent setting, where non-congested traffic conditions are assumed throughout the day throughout the network, is attempted. However, the acknowledgement of the fact that travel speed could not be freely chosen in congested urban areas, as it fully depends on the expected time-varying traffic conditions, has led to the consideration of time-dependent variants of the VRP for a more accurate estimation model of fuel and other relevant decisions with temporal dependencies (Figliozzi, 2010; Figliozzi, 2011; Franceschetti, Honhon, Woensel, Bektas, \& Laporte, 2013; Ehmke et al., 2016; Androutsopoulos \& Zografos, 2017; Çimen \& Soysal, 2017; Ehmke et al., 2018). Some of these studies also consider the possible benefits of waiting at the depot and/or the customers (Xiao et al., 2012; Franceschetti et al., 2013; Androutsopoulos \& Zografos, 2017).

Very recent research (Turkensteen, 2017; Kancharla \& Ramadurai, 2018) has shed light on the inaccuracy of the fuel consumption estimation model used within EMVRPs due to ignoring truck A/D rates. Using numerical experiments from available chassis dynamometer driving schedules, Turkensteen (2017) shows that the magnitude of this error can be high. Kancharla and Ramadurai (2018), on the other hand, collect some on-road truck A/D data in a time-independent and static setting and randomly feed these data into their model for fuel consumption estimation. A major shortcoming of their proposed approach, however, lies in the fact that the spatial and temporal characteristics of the road-links in the graph are completely ignored. This issue is addressed in the current work by using the microscopic CMEM formula that incorporates instantaneous time-dependent second-by-second speed and A/D rates.

The effect of the type and the number of the trucks that are included in the fleet to execute the routes on fuel consumption was previously considered in the context of the EMVRPS by Koç et al. (2014) and Xiao and Konak (2016). The studies of Demir, Bektaş, and Laporte (2014) and Androutsopoulos and Zografos (2017) are the only available studies that identify the objectives of fuel consumption minimisation and driving time as two conflicting objectives and study the problem as a bi-objective optimisation problem. Some very recent research work has also acknowledged the problem of fuel-consumption minimising path identification in a time-dependent setting, and new emissions minimising vehicle routing models considering alternative road-paths between the consecutive truck stops have been published very recently (Qian \& Eglese, 2016; Ehmke et al., 2016; Huang et al., 2017; Androutsopoulos \& Zografos, 2017). This last category of research is the most pertinent to the current study and will be discussed further in the sequel.

In Table 1 different attributes and features that are considered by the key literature in the area and the current work are indicated using tick marks. This table can highlight two major gaps in the field that the proposed work is trying to address: (i) despite its important implications with regard to multiple use of energy-efficient resources multiple times during 
the planning horizon, multi-trips decision making has not yet been incorporated into emissions minimising vehicle routing models, and (ii) all factors and attributes identified and addressed have not yet been unified into a realistic integrated modelling and solution framework. It is also worth mentioning that the proposed work in this paper is the first study in the area to consider vehicle cost as a major business objective next to makespan and fuel consumption objectives in a triobjective setting.

Table 1

Overview of attributes covered by the previous related works

\begin{tabular}{|c|c|c|c|c|c|c|c|c|c|c|}
\hline \multirow[b]{2}{*}{ Study } & \multicolumn{10}{|c|}{ Attributes covered } \\
\hline & 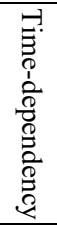 & 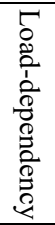 & 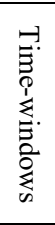 & 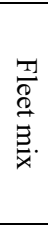 & 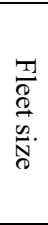 & 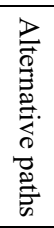 & 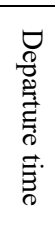 & 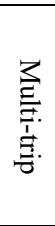 & 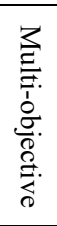 & 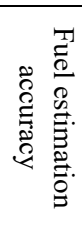 \\
\hline Kara et al. (2007) & $x$ & $\checkmark$ & $x$ & $x$ & $x$ & $x$ & $x$ & $x$ & $x$ & $x$ \\
\hline Ubeda et al. (2011) & $x$ & $\checkmark$ & $x$ & $x$ & $x$ & $x$ & $x$ & $x$ & $x$ & $x$ \\
\hline Bektas and Laporte (2011) & $x$ & $\checkmark$ & $\checkmark$ & $x$ & $x$ & $x$ & $x$ & $x$ & $x$ & $x$ \\
\hline Figliozzi (2011) & $\checkmark$ & $x$ & $\checkmark$ & $x$ & $x$ & $x$ & $x$ & $x$ & $x$ & $x$ \\
\hline Franceschetti et al. (2013) & $\checkmark$ & $\checkmark$ & $\checkmark$ & $x$ & $x$ & $x$ & $x$ & $x$ & $x$ & $x$ \\
\hline Demir et al. (2014) & $x$ & $\checkmark$ & $\checkmark$ & $x$ & $x$ & $x$ & $x$ & $x$ & $\checkmark$ & $x$ \\
\hline Koç et al. (2014) & $x$ & $\checkmark$ & $\checkmark$ & $\checkmark$ & $\checkmark$ & $x$ & $x$ & $x$ & $x$ & $x$ \\
\hline Xiao and Konak (2016) & $\checkmark$ & $\checkmark$ & $\checkmark$ & $\checkmark$ & $\checkmark$ & $x$ & $\checkmark$ & $x$ & $x$ & $x$ \\
\hline Qian and Eglese (2016) & $\checkmark$ & $x$ & $\checkmark$ & $x$ & $x$ & $\checkmark$ & $x$ & $x$ & $x$ & $x$ \\
\hline Ehmke et al. (2016) & $\checkmark$ & $\checkmark$ & $x$ & $x$ & $x$ & $\checkmark$ & $x$ & $x$ & $x$ & $x$ \\
\hline Huang et al. (2017) & $\checkmark$ & $\checkmark$ & $\checkmark$ & $x$ & $x$ & $\checkmark$ & $\checkmark$ & $x$ & $x$ & $x$ \\
\hline Androutsopoulos and Zografos (2017) & $\checkmark$ & $\checkmark$ & $\checkmark$ & $x$ & $x$ & $\checkmark$ & $\checkmark$ & $x$ & $\checkmark$ & $x$ \\
\hline Kancharla and Ramadurai (2018) & $x$ & $\checkmark$ & $x$ & $\checkmark$ & $x$ & $x$ & $x$ & $x$ & $x$ & $\checkmark$ \\
\hline Proposed work & $\checkmark$ & $\checkmark$ & $\checkmark$ & $\checkmark$ & $\checkmark$ & $\checkmark$ & $\checkmark$ & $\checkmark$ & $\checkmark$ & $\checkmark$ \\
\hline
\end{tabular}

Except for the last category of the models discussed above (i.e. models that consider alternative road-paths), a major limitation of most of the existing research work in the area of EMVRPs lies in the fact that they consider an a priori determined single road-path for travelling between each pair of customers. There are at least two main reasons why this is not possible when routing on a congested urban road network for fuel consumption minimisation:

(1) Determining a minimum fuel consuming path between a given pair of origin/destination on urban road networks with time-varying traffic conditions requires a knowledge of the time the origin node is to be departed, the type of the truck to be dispatched to traverse the path (in case of a heterogeneous fleet), and the load to be carried by the dispatched truck over the path. All these variables are unknown until the routing plan and schedule is fully realised, and therefore identifying a path (or a set of paths) between every pair of the required nodes (customers and the depot) in order to transform the roadway network into a complete graph seems impossible.

(2) For a given sequence of visits starting and terminating at the depot (a vehicle route), and a given departure time from the depot, it is not guaranteed that merely fuel consumption minimising paths are taken by the truck between every pair of consecutive stops in order to minimise the total amount of fuel required by the vehicle route. In other words, inferior paths in terms of the fuel consumption might appear in the optimal fuel consuming vehicle route (see examples in Androutsopoulos and Zografos - 2017). 
We are aware of 4 papers that acknowledge one or both of these issues and try to address them. Qian and Eglese (2016) and Huang et al. (2017) propose to use a multi-graph of the Time-Dependent Shortest Paths (TDSPs) between the required nodes, where a set of such paths as candidate paths are precomputed between every pair of required nodes and kept. However, this approach is not sufficient as it does not take load (and vehicle type) dependency into account. In the hope of partially tackling the effect of load-dependency, Huang et al. (2017) also include the distance-minimising path to the set of the time-minimising paths. While this might be partially helpful, there is no guarantee that all eligible road-paths are included. Ehmke et al. (2016) identify the first issue mentioned above and state that the identification of a set of all eligible paths a priori is not possible. Instead, to take both time and load-dependency into consideration, they propose a new result that identifies a condition under which a time-dependent path between two customers is load invariant (in case of a homogenous fleet). This allows them to reduce the computational challenge of finding the time and load-dependent paths between some customers at some time instants by making it possible to precompute expected time-dependent fuel consumption minimising paths between them. However, still for the rest of the customer pairs where the condition they check is not satisfied they need to carry out shortest path computation on-the-fly in their Tabu Search algorithm, which is a costly requirement that prohibits solving problems with larger than 30 customers even heuristically. Androutsopoulos and Zografos (2017) acknowledge both of the stated issues and propose a network reduction approach that is based on the use of the $k$-shortest distance road paths. This approach is, however, sensitive to the selection of the value of $k$. They try to show that when $k$ is small (e.g. $k=2$ ) eligible paths might be excluded from the reduced network, and if a higher value for $k$ is selected (e.g. $k=5$ ), while the number of excluded eligible paths is reduced, the computational time increases, accordingly.

Based on this review, the exact identification of the full set of the eligible emissions minimising road-paths between the required nodes on a time-dependent graph is still an open research issue. To tackle this, an efficient exact Path Elimination Procedure is proposed by this paper that advances the result found by Ehmke et al. (2016) and can identify and discard all proven to be redundant paths between the required nodes in a pre-processing stage and eliminates the need for the shortest-path calculation on-the-fly. Our results are generalised for the case of a heterogeneous fleet, with multiple objective functions to be minimised by the planned routes.

\section{Model development: notation and definitions}

The SPRP is defined on a directed graph $G=(N, A)$, representing a real roadway network, where $N$ is the set of network nodes and $A$ is the set of directed road-links. The set $N=\left\{N_{0} \cup N_{1} \cup N_{2}\right\}$ is comprised of the depot $N_{0}=\{0\}$, customer nodes $N_{1}=\{1,2, \ldots, n\}$, and network junctions $N_{2}=\{n+1, \ldots, n+m\}$. There is a fleet of heterogeneous vehicles $K$, with $|K|=\vartheta$, located in the central depot, which is assumed to be composed of $\mathbb{k}$ different types of trucks. To each truck $k \in K$ a curb weight $\mu_{k}(\mathrm{~kg})$, a maximum payload $Q_{k}(\mathrm{~kg})$, and a daily hiring fixed cost $c_{k}(£)$, among other vehiclespecific factors such as engine friction factor, engine speed, engine displacement, coefficient of aerodynamic drag, and frontal surface area is attributed.

Each customer $i \in N_{1}$ is associated with a certain demand $q_{i} \leq \max _{k \in K} Q_{k}$ to be delivered within its pre-determined hard time window denoted by $w_{i}=\left[e_{i}, l_{i}\right]$, with service time $s_{i}$. The depot working hours which is also considered as the planning horizon is denoted by $T=w_{0}=\left[e_{0}, l_{0}\right]$, and while it is assumed that trucks are initially loaded, reloading them for operating a new route takes $s_{0}$ time at the depot. To each road-link $(i, j) \in A$, a distance $d_{i j}$, and a time-dependent 
travel time $t_{i j}^{\tau}$, depending on the departure time from the origin node $i$, i.e. $\tau \in\left[e_{0}, l_{0}\right]$ is attributed. In this study we assume that the time-dependent travel times $\left(\boldsymbol{t}_{i j}^{\tau}\right)$ are integer.

The aim of the SPRP is to determine an optimal composition of vehicles in the fleet to operate routes that start and finish at the depot and serve every customer exactly once within their pre-defined time-windows, without violating vehicle capacities and working day limits, such that the following objectives are minimized: (i) vehicle hiring cost, (ii) total amount of fuel consumed, and (iii) total makespan (duration) of the routes.

The following terms are used throughout this paper:

- Required nodes: required nodes $\left(N_{R}\right)$ are the nodes on the roadway network representing the location of the depot and the customers; i.e. $N_{R}=N_{0} \cup N_{1}$.

- Road-link: a road-link is any kind of road in the hierarchy of roads such as a freeway, an arterial, a collector, or a local road that connects two nodes on the roadway network; i.e. $(i, j) \in A$.

- Road-path: a road-path $p_{i j}$, or simply a path, is a sequence of road-links which connects a pair of required nodes $i, j \in N_{R}, i \neq j$ on the roadway network; i.e. $p_{i j}=[(i, 1),(1,2), \ldots,(\ell, j)], 1 \ldots \ell \in N \backslash i, j$. By convention, let us assume that $p_{i j}$ is the set of all paths between a pair of required nodes, i.e. $p_{i j}=\left\{p_{i j, 1}, p_{i j, 2}, \ldots, p_{i j, \ell}\right\}$ (identification of this set can be intractable). Whenever it is needed, we denote the time-dependent travel time of a road-path $p_{i j}$ by $t^{\tau}\left(p_{i j}\right)$ for departure time $\tau$ from node $i$; moreover, the fuel consumption of a truck $k \in K$, carrying a load $f \in\left[0, Q_{k}\right]$ over a given road-path $p_{i j}$ is denoted by $\mathfrak{f}_{f}^{k \tau}\left(p_{i j}\right)$. Note that since no waiting is allowed at intermediate nodes between the origin and the destination of the path, knowing the departure time from the origin node is sufficient for estimating the time-dependent attributes of the path.

- Route (trip): A route or a vehicle trip $(r)$ is a sequence of visits starting at the depot, passing through at least one customer and terminating at the depot, i.e. $r=\{0, i, \ldots, 0\}, i \in N_{1}$.

- Route-path: A route-path $\left(r_{p}\right)$ of route $(r)$ is a route enhanced by the road-paths connecting every pair of consecutive required nodes on the route; i.e. $r_{p}=\left\{\left(0, i, p_{0 i, 1}\right), \ldots,\left(j, 0, p_{j 0,1}\right)\right\}, i, j \in N_{1}, p_{0 i, 1} \in p_{0 i}, p_{0 j, 1} \in$ $p_{j 0}$.

○ Route-trajectory: A route-trajectory $\left(r_{p}^{\tau_{0}}\right)$ is basically a scheduled route-path detailing the departure time from the depot and hence each required node on the route-path, i.e. $r_{p}^{\tau_{0}}=\left\{\left(0, i, p_{0 i, 1}, \tau_{0}\right), \ldots,\left(j, 0, p_{j 0,1}, \tau_{\ell}\right)\right\}, i, j \in$ $N_{1}, p_{0 i, 1} \in p_{0 i}, p_{0 j, 1} \in p_{j 0}, \tau_{0}, \tau_{\ell} \in T$. The total makespan and fuel consumption of a truck $k \in K$ over a routetrajectory $r_{p}^{\tau_{0}}$ are denoted by $t\left(r_{p}^{\tau_{0}}\right)$ and $\mathfrak{f}^{k}\left(r_{p}^{\tau_{0}}\right)$, respectively. Note that, since $\mathfrak{f}^{k}\left(r_{p}^{\tau_{0}}\right)$ is deduced from the aggregation of the fuel consumption over each of the constituting road-paths with varying payloads, it is not indexed by the truck load.

- SPRP solution: A feasible SPRP solution is a set of feasible route-trajectories enhanced by the type of the truck with enough capacity to carry out each one of them. The solution moreover specifies the amount of load that is carried by each truck over each road-path, and any need for reloading any of the vehicles for extra rounds of trip.

The SPRP is categorised as a Multi-Objective MILP (MOMILP) problem with three conflicting objectives. Unlike the single objective optimisation, where a global optimal solution is reachable, solution to an MOMILP is the set of the ND points, or efficient solutions called the Pareto Optimal Set (POS). The reader is referred to Coello, Lamont, and Van 
Veldhuizen (2007) for definitions relevant to Pareto optimality, Pareto dominance, weak/strict Pareto optimality and the Pareto front.

A list of all notation and acronyms used throughout the paper is provided in Appendix A of the paper.

\subsection{The time-dependent travel time estimation model}

Most of the existing research work in the area of the Time-Dependent VRP (TDVRP) relies on the model proposed by Ichoua, Gendreau, and Potvin (2003) for the calculation of the time-dependent travel time of a road-link. Their algorithm uses a step function for speed to deduce a piecewise linear function for travel time that satisfies the FIFO property. However, despite its ease of use, the application of the model proposed by Ichoua et al. (2003) on real life congestion situations is hindered by their extra simplification in viewing congestion speed as a step function which implies that changes in travel speed occur instantly (i.e. A/D rates equal to infinity) with unjustified leaps from one level to the next, ignoring the time required for A/D. In reality, a lot of such A/Ds occur during the actual arc traversal, especially in congested urban areas, and this leads to lack of accuracy in estimating the expected travel times.

This shortcoming can be overcome by using the travel time model in Horn (2000) which allows using directly the archived point-based historical speed data. Connecting each speed observation at each given time instant results in a continuous piecewise linear function of the time for speed (including A/Ds) (Figure 1), using which a FIFO-consistent nonlinear travel-time function could be deduced. In Horn (2000) the computation of these travel times is performed by counting time from the departure time up to the point in time that a distance equal to the length of the arc is traversed, which can be computationally intensive. Androutsopoulos and Zografos (2012) enhance this model by presenting a closed form formula for calculating the travel time on any road-link given a departure time from the origin node, based on the computation of the arrival time at the destination node. Here, we propose an alternative closed form approach that compared with that of Androutsopoulos and Zografos (2012) is less complicated to implement and use, and unlike their formula does not require to observe if departure and arrival time occur within the same time interval or not. Another added advantage of the proposed formula is that it could be simply used in backward travel time calculation (i.e. finding the departure time for an intended arrival time).

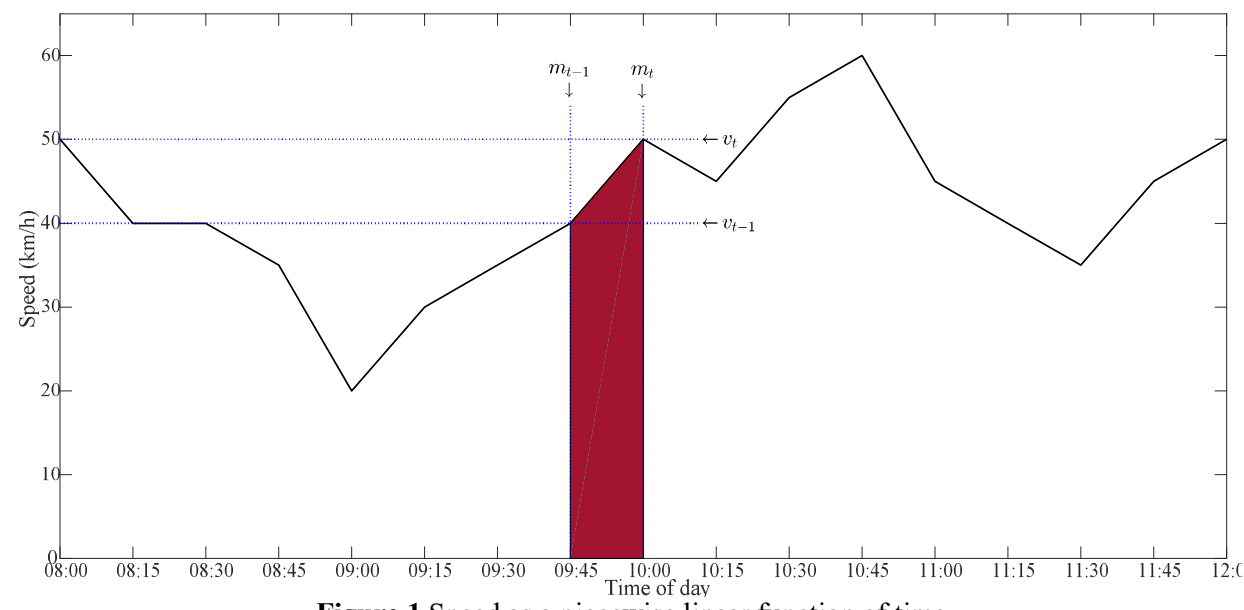

Figure 1 Speed as a piecewise linear function of time

To deduce the intended closed-form formula, suppose that there are $h_{i j}$ speed observations during the planning horizon $T$ for road-link $(i, j) \in A$. For notational simplicity, in the rest of this section we drop $i j$ indices for all parameters. We denote by $v_{t}, t \in\{1, \ldots, h\}$ the speed observation at time instant $m_{t}$. The line segment connecting $v_{t}$ to $v_{t+1}$ has a slope $a_{t}$ (i.e. A/D rate) equal to $\left(v_{t+1}-v_{t}\right) /\left(m_{t+1}-m_{t}\right)$, and an intercept $\beta_{t}$ equal to $\left(v_{t}-a_{t} m_{t}\right), \forall t \in\{1, \ldots, h-1\}$ 
$\left(a_{t}=\beta_{t}=0\right.$ for $\left.t=h\right)$. The distance that could be traversed from time instant $m_{1}=e_{0}$ until time instant $m_{t}, t \in$ $\{2, \ldots, h\}$, is denoted by $\delta_{t}$, and could be calculated as follows:

$$
\delta_{t}=\delta_{t-1}+\left[\frac{1}{2}\left(m_{t}-m_{t-1}\right)\left(v_{t}+v_{t-1}\right)\right], \quad \delta_{1}=0, t \in\{2, \ldots, h\}
$$

This equation is based on the premise that the area below the speed curve is equal to the physical distance that can be travelled. The equation hence calculates sequentially the area below the speed curve bounded by each pair of consecutive time instants (which is indeed a right trapezoid as highlighted in Figure 1) and adds this area to the entire area below the curve up until time instance $m_{t-1}$, i.e. $\delta_{t-1}$. Based on the definition of $\delta_{t}$, the time-definitive accumulated distance function $\varsigma$, which is the building block of our closed form formula, is defined as follows:

Definition 1 The time-definitive accumulated distance function $\varsigma(\tau)$ is defined as a function that calculates the distance possible to traverse from the beginning of the planning horizon until any given time instant $\tau \in T$ :

$$
\varsigma(\tau)=\delta_{t+1}-\left[\frac{1}{2}\left(m_{t+1}-\tau\right)\left(v_{\tau}+v_{t+1}\right)\right], \quad \tau \epsilon\left[m_{t}, m_{t+1}\right), t \in\{1, \ldots, h-1\}
$$

where $v_{\tau}$ denotes the speed at time instant $\tau$, which is equal to $v_{t}+\left[a_{t}\left(\tau-m_{t}\right)\right]$.

Then, for any given road-link in the network with distance $d$ the following relationship always holds: $d=\varsigma\left(\tau_{\varpi}\right)-$ $\varsigma\left(\tau_{\mathbb{d}}\right)$, where $\tau_{\mathbb{d}}$ denotes the departure time from the origin of the given road-link, and $\tau_{\llbracket}$ denotes the arrival time at the destination of the road-link. Hence, for any given departure time $\tau_{\mathbb{d}}$, the arrival time $\tau_{\llbracket}$ could be found using the inverse of the $\varsigma$ function, and this implies the possibility of proposing a FIFO-consistent closed form formula for the time-dependent travel time calculation. To derive such formula, as $d=\varsigma\left(\tau_{\varpi}\right)-\varsigma\left(\tau_{d}\right)$, we have $\varsigma\left(\tau_{\varpi}\right)=d+\varsigma\left(\tau_{d}\right)$, which can be written as $\varsigma\left(\tau_{\varpi}\right)=E$, where $E=d+\varsigma\left(\tau_{d}\right), E \in\left[\delta_{t}, \delta_{t+1}\right), t \in\{1, \ldots, h-1\}$. Then, based on the definition of $\varsigma$ function we can write: $\delta_{t+1}-\left[\frac{1}{2}\left(m_{t+1}-\tau_{\varpi}\right)\left(v_{\tau_{\bullet}}+v_{t+1}\right)\right]=E$, and further based on the definitions of $v_{\tau_{\bullet}}$ and $\delta_{t+1}$, we can write: $\delta_{t}+$ $\left[\frac{1}{2}\left(m_{t+1}-m_{t}\right)\left(v_{t+1}+v_{t}\right)\right]-\left[\frac{1}{2}\left(m_{t+1}-\tau_{\varpi}\right)\left(a_{t} \tau_{\varpi}+\beta_{t}+v_{t+1}\right)\right]=E$, and writing this for $\tau_{\varpi}$ we will have the following expression:

$$
\tau_{\square}= \begin{cases}\frac{\left[\beta_{t}^{2}-2 a_{t} \delta_{t}+2 a_{t} E+a_{t}^{2} m_{t}^{2}+2 a_{t} m_{t} \beta_{t}\right]^{1 / 2}-\beta_{t}}{a_{t}}, & a_{t} \neq 0 \\ m_{t}+\frac{E-\delta_{t}}{v_{t}}, & a_{t}=0\end{cases}
$$

Note that the model by Ichoua et al. (2003) is a special case of expression (3), where $a_{t}=0, \forall t \in T$. Also note that this formula can use microscopic traffic speed data (i.e. second-by-second speed variations) as well as macroscopic data (e.g. every 5, 10, or 15 minutes) as input. As will be discussed later in section 6 of the paper, when microscopic data are not available, the travel time estimated from macroscopic data using this formula provides a basis for the generation of synthetic driving cycles with instantaneous speed variations.

For the most efficient implementation of (3), all model parameters including $a_{t}, \beta_{t}$, and $\delta_{t}$, and also $\varsigma(\tau)$ and $v_{\tau}$ for all possible departure times, could be pre-computed, which then make the application of expression (3) pretty simple and straightforward. A useful feature of (3) is that it is also possible to find the departure time $\tau_{\mathbb{d}}$ for any given arrival time $\tau_{\llbracket}$ using the same formula with the only modification that $E=\varsigma\left(\tau_{\varpi}\right)-d$.

It is worth mentioning that the time-dependent travel time of a given scheduled road-path could be simply estimated from its constituent road-links, and thus as the time-dependent travel times of the road-links preserve the FIFO property, any simple paths considered on the graph would be also FIFO-consistent (given that waiting is not allowed on intermediate nodes). 


\subsection{The instantaneous fuel consumption estimation model}

Assume that the spatiotemporal instantaneous driving cycles $D C_{i j}^{\tau}$, denoting the expected second-by-second speed variations, are available for each road-link $(i, j) \in A$ of the network, for all time instants $\tau \in T$. It is worth mentioning that with the current advancements in the Global Positioning System (GPS) devices, it is possible to create a historical archive of such data for the required road-links at different times of a day (Belliss, 2004; Byon, Shalaby, \& Abdulhai, 2006; Lee, Sener, \& Mullins III, 2016); however, in the event that they are unavailable at the planning stage, they could be instead generated synthetically using the approach proposed later in the paper.

Given such cycles, the instantaneous time, load and truck-type dependent fuel consumption (in litres) over the given road link $(i, j) \in A$ for vehicle $k \in K$, i.e. $\mathcal{F}_{i j}^{k \tau}$, could be computed using the CMEM formula of Barth et al. (2004) as follows:

$$
\begin{gathered}
\mathcal{F}_{i j}^{k \tau}=\sum_{t=\tau}^{\tau+t_{i j}^{\tau}} \frac{\zeta}{\kappa \psi}\left(H_{k} \mathcal{N}_{k} V_{k}+\frac{0.5 C_{k} \rho A_{k} v_{i j t}^{3}}{1000 \varepsilon \varpi}\right)+\left(\mu_{k}+f_{i j}\right) \sum_{t=\tau}^{\tau+t_{i j}^{\tau}} \frac{\zeta}{\kappa \psi}\left(\frac{v_{i j t}\left(g \sin \theta+g C_{r} \cos \theta+a_{t}\right)}{1000 \varepsilon \varpi}\right), \\
\forall(i, j) \in A, k \in K, \tau \in T
\end{gathered}
$$

where $\zeta$ is fuel-to-air mass ratio, $\kappa$ is the heating value of a typical diesel fuel $(\mathrm{kJ} / \mathrm{g}), \psi$ is a conversion factor from grams to litres (from $(g / s)$ to $(l / s)), H_{k}$ is the engine friction factor $(k J / r e v / l)$ for vehicle $k, \mathcal{N}_{k}$ is the engine speed (rev/s) for vehicle $k, V_{k}$ is the engine displacement ( $l$ ) for vehicle $k, \rho$ is the air density $\left(\mathrm{kg} / \mathrm{m}^{3}\right), A_{k}$ is the frontal surface area $\left(\mathrm{m}^{2}\right)$ for vehicle $k, v_{i j t}$ is the vehicle speed $(\mathrm{m} / \mathrm{s})$ at the $t$ th second of the cycle, $\mu_{k}$ is the vehicle curb weight $(k g)$ for vehicle $k, f_{i j}$ is the load $(\mathrm{kg})$ carried over the given road link by the truck, $g$ is the gravitational constant (equal to $9.81 \mathrm{~m} / \mathrm{s}^{2}$ ), $\theta$ is the road angle, $C_{k}$ and $C_{r}$ are the coefficient of aerodynamic drag and rolling resistance, $\varepsilon$ is vehicle drive train efficiency and $\varpi$ is an efficiency parameter for diesel engines.

Expression (4) divides CMEM into a time-dependent term Unrelated to Truck Mass (called the UTM attribute and indicated by $Z_{i j}^{k \tau}$ hereafter), and a time-dependent term linearly Related to the Truck Mass (called the RTM attribute and indicated by $\Gamma_{i j}^{\tau}$ hereafter), and both of these could be precomputed and stored for all road-links (or road-paths) at all possible departure times based on the available $D C_{i j}^{\tau}$ s. Hence, this expression could be simply re-written as $\mathcal{F}_{i j}^{k \tau}=Z_{i j}^{k \tau}+$ $\Gamma_{i j}^{\tau}\left(\mu_{k}+f_{i j}\right), \forall(i, j) \in A, \tau \in T$ (to see more detail on the derivation of this formula in a homogenous fleet case, the reader can refer to appendix A in Androutsopoulos and Zografos - 2017). As a note on the storage space requirement for storing all UTM and RTM attributes along with time-dependent travel times for all road-links at all possible departure times, it should be mentioned that the space complexity is $O((\mathbb{k}+2)|A||T|)$, where $|A|$ is the number of network roadlinks, and $|T|$ is the number of all possible departure times. However, as will be explained later in section 4.1., this required storage space could be critically reduced by using 'time periods' instead of 'time instants'.

In this study, for experimental purposes, similar to the work of Koç et al. (2014) on the fleet size and mix PRP, we consider the fleet to be composed of light, medium and heavy duty trucks and use the same values they use for the common and vehicular specific parameters, which they obtain for the three main vehicle types of MAN Trucks (see Tables 1 and 2 in Koç et al. - 2014).

\section{The Path Elimination Procedure (PEP)}

The intention of this section is to deal with an important prerequisite to any subsequent exact/heuristic solution algorithm for the SPRP, which is to alleviate the difficulty of solving the problem directly on the real roadway network, without losing the essential information contained in the original graph. As discussed in section 2 of the paper, existing approaches 
in the literature can only identify a limited subset of eligible road-paths that must be preserved between the required nodes and cannot guarantee that all paths that might be used in the design of an optimal vehicle route are identified and preserved. In the case of the SPRP, a much more complicated situation must be coped with, since not only a time, load and truck type dependent fuel consumption objective is to be minimised, but also this objective is considered alongside two other conflicting objectives, and any set of the paths that are returned by any pre-processing algorithm should ensure that ad-hoc ND points will not be eliminated.

Since the main problematic objective function that causes complications is the time, load and truck type dependent fuel minimisation objective, we begin by focusing on this objective only, and then generalise all our results for the multiobjective case of the SPRP. The underlying idea of the proposed PEP in this section is hence to identify and retain all roadpaths that might be used by at least one of the truck types in the fleet, for at least one time instant during the planning horizon, to carry some load levels in the range of the truck capacity, and then eliminate all other paths as redundant paths from the network. An "eligible" path can be therefore defined formally as follows:

Definition 2 A road-path $p_{i j, a}$ between a pair of required nodes $i, j \in N_{R}: i \neq j$ is called an 'eligible' path, iff $\exists k \in$ $K, f \in\left[0, Q_{k}\right], \tau \in T: \mathfrak{f}_{f}^{k \tau}\left(p_{i j, a}\right) \leq \mathfrak{f}_{f}^{k \tau}\left(p_{i j, \ell}\right), \forall p_{i j, \ell} \in p_{i j}$.

The elimination of an eligible road-path from the underlying roadway network can hence lead to a suboptimal vehicle route in terms of fuel consumption, and all such paths must be identified and retained.

In order to set the scene, the motivation of the PEP is reiterated through the following remarks:

Remark 1 Determining a priori a (set of) minimum fuel consuming road-path(s) between a given pair of origin/destination on urban road networks with time-varying congestion seems impossible.

Remark 2 A minimum fuel consuming route-trajectory is not necessarily concatenated; i.e. its constituent scheduled roadpaths are not necessarily optimal, and they can be road-paths which are inferior in terms of the fuel consumption.

It is easy to acknowledge the first remark, which stems from our lack of knowledge about the departure time from the origin node, the type of the truck that is going to traverse the path and the amount of load that the truck is going to carry over the road-path, prior to realising the full truck route and schedule. However, the second remark is not as intuitive, because it might seem that once fuel consumption minimising road-paths between every consecutive visit, for every possible departure time, and any load on the trucks are known, these paths can be retained to minimise the overall amount of fuel required by the route, and the alternative inferior road-paths could be simply ignored. However, it is not difficult to show that it might be beneficial to take road-paths that are not optimal in terms of fuel consumption to gain improvements in the overall fuel consumption of the route (see example 1 in Androutsopoulos and Zografos - 2017). Note that despite Androutsopoulos and Zografos (2017) identify this as an inherent issue for the bi-objective time-dependent VRPs, it is even an issue in a single objective case. In fact, this is a largely ignored situation in any general routing for some timedependent cost minimisation in a time-dependent network, and an important generalisation of Remark 2 is that the cheapest path in a time-dependent setting is not necessarily concatenated.

Let $\mathcal{D}_{i j}$ be the set of all minimum fuel consuming paths between required nodes $i$ and $j$ for all possible departure times $\tau \in T$ from node $i$. Then, building on some previous results for the time-dependent shortest path problems (Orda \& Rom, 1990; Hamacher, Ruzika, \& Tjandra, 2006) the following theorem is proposed: 
Theorem 1 Suppose the set $\mathcal{E}_{i j}$ is the set of all paths $p_{i j}$ with non-dominated vectors $\left[\boldsymbol{t}^{\tau}\left(p_{i j}\right), \mathfrak{f}_{f}^{k \tau}\left(p_{i j}\right)\right]$ for at least one $\tau \in T, k \in K$, and $f \in\left[0, Q_{k}\right]$ (note that $\mathcal{D}_{i j} \subseteq \mathcal{E}_{i j}$ ), and let $\mathcal{E}=\left\{\mathcal{E}_{i j} \mid i, j \in N_{R}, i \neq j\right\}$; if departure time from the depot is unrestricted, then any optimal route-trajectory in terms of fuel consumption has its road-paths in $\mathcal{E}$.

Proof. Without loss of generality, assume that $s_{i}=0$ and $w_{i}=w_{0}$ for all $i \in N_{1}$. With this assumption, the departure time from a required node is upon the arrival time at the node from an upstream required node. Suppose that $r_{1}^{\tau_{0}}=$ $\left\{\left(0, i, p_{0 i, 1}, \tau_{0}\right),\left(i, 0, p_{i 0,1}, \tau_{1}\right)\right\}$ is an optimal route-trajectory in terms of fuel consumption. Since there is only one customer $i \in N_{1}$ that is served over this route-trajectory, a truck $k \in K$, that is sufficiently large to carry $q_{i}$ is used. According to the backward principle of optimality (see Definition 3.2 and Theorem 3.2 in Hamacher et al. - 2006) roadpath $p_{i 0,1}$ is optimal in terms of fuel consumption for departure time $\tau_{1}$ from node $i$, and thus $p_{i 0,1} \in \mathcal{E}$. Therefore, we must only prove that $p_{0 i, 1} \in \mathcal{E}$. To use a proof by contradiction, initially suppose that $p_{0 i, 1}$ is not in $\mathcal{D}_{0 i}$. The assumption that $p_{0 i, 1}$ is not in $\mathcal{D}_{0 i}$ implies that there is a fuel consumption minimising path $p_{0 i, 2} \in \mathcal{D}_{0 i}$ that arrives at node $i$ at time $\tau_{1}$. Assume that to arrive at customer $i$ at time $\tau_{1}$, the truck must depart the origin of path $p_{0 i, 2}$ (i.e. the depot) at time $\tau_{2}$ (remember that departure time from the depot is not restricted). This means $f_{q_{i}}^{k \tau_{2}}\left(p_{0 i, 2}\right)<\mathfrak{f}_{q_{i}}^{k \tau_{0}}\left(p_{0 i, 1}\right)$, and there is a routetrajectory $r_{2}^{\tau_{2}}=\left\{\left(0, i, p_{0 i, 2}, \tau_{2}\right),\left(i, 0, p_{i 0,1}, \tau_{1}\right)\right\}$, such that $f^{k}\left(r_{2}^{\tau_{2}}\right)<f^{k}\left(r_{1}^{\tau_{0}}\right)$; contradicting the fact that $r_{1}^{\tau_{0}}$ is an optimal route trajectory. This proof is, however, incomplete under a certain condition; the departure time from the depot for path $p_{0 i, 2}$, i.e. $\tau_{2}$ can be smaller or larger than $\tau_{0}$, meaning that $t^{\tau_{0}}\left(p_{0 i, 1}\right)$ can be smaller or larger than $t^{\tau_{2}}\left(p_{0 i, 2}\right)$. Under the condition that $\tau_{2}<\tau_{0}$ and $\tau_{2}<e_{0}$, path $p_{0 i, 2}$ is infeasible; however, as in that case $p_{0 i, 1}$ has a non-dominated vector $\left[\boldsymbol{t}^{\tau_{0}}\left(p_{0 i, 1}\right), \mathfrak{f}_{q_{i}}^{k \tau_{0}}\left(p_{0 i, 1}\right)\right]$, it already exists in $\mathcal{E}$.

Based on this theorem, the key to address the situation in Remark 2 is indeed departure time optimisation, and except for a special case, working on a graph based on $\mathcal{D}$ is usually sufficient for the minimisation of the fuel consumption by the routes. However, for completeness this theorem proposes to work on $\mathcal{E}$, since if the set $\mathcal{E}$ could be somehow constructed, the same minimum fuel consuming route-trajectories that can be found directly on $G$ could be found on $\mathcal{E}$. In the sequel, we propose new results to construct this set.

In the rest of this section, whenever we refer to a road-path, it is meant to be a road-path between a given pair of required nodes $i, j \in N_{R}$, but for notational simplicity we drop origin/destination indices of paths and their attributes. Moreover, instead of writing $t^{\tau}\left(p_{\mathfrak{p}}\right), Z^{k \tau}\left(p_{\mathfrak{p}}\right)$ and $\Gamma^{\tau}\left(p_{\mathfrak{p}}\right)$ for the attributes of a path $p_{i j, \mathfrak{p}} \in p_{i j}$, we simply write $t_{\mathfrak{p}}^{\tau}, Z_{\mathfrak{p}}^{k \tau}$ and $\Gamma_{\mathfrak{p}}^{\tau}$, respectively.

Proposition 1 (Ehmke et al., 2016) If path $p_{1}$ is a fuel consumption minimising path for both a fully loaded truck of type $k$, and an empty truck of the same type $k$ for departure time instant $\tau$, then this path is optimal in terms of fuel consumption for any other size of load on the truck of type $k$.

This proposition modifies slightly the proposition stated in Ehmke et al. (2016), in the sense that they do not explicitly state that this is a condition that must be checked for all possible departure times. Moreover, to generalise it for a heterogeneous fleet, the type of the truck matters and is mentioned here. It is also worth noting that while they have proposed this proposition in the context of the average-speed CMEM, their proof is applicable for the case of the instantaneous CMEM, as well. 
While Proposition 1 establishes an interesting result, which can be used to precompute expected time-dependent fuel consumption minimising paths between some customers at some time instants, our computational experiments on a real world urban road network demonstrate that there are cases when this condition is not satisfied for up to around $60 \%$ of the times (see Appendix B); nevertheless, Proposition 1 serves as a building block to a more important theorem that underpins the proposed PEP:

Theorem 2 If for a given departure time $\tau$, path $p_{1}$ is a fuel consumption minimising path for a fully loaded truck of type $k$, and path $p_{2}$ is a fuel consumption minimising path for an empty truck of the same type $k$ such that $p_{1} \not \equiv p_{2}$, then any other path $p_{a}$ is an eligible path iff it is a fuel consumption minimising path for truck type $k$ carrying some load $f \in$ $\left[\frac{Z_{a}^{k \tau}-Z_{2}^{k \tau}}{\Gamma_{2}^{\tau}-\Gamma_{a}^{\tau}}-\mu_{k}, \frac{Z_{1}^{k \tau}-Z_{a}^{k \tau}}{\Gamma_{a}^{\tau}-\Gamma_{1}^{\tau}}-\mu_{k}\right]$

Proof. We first lay out some useful valid inequalities derived from the assumptions:

The optimality of $p_{1}$ for the fully loaded truck yields the following inequalities:

$$
\begin{aligned}
& Z_{1}^{k \tau}+\Gamma_{1}^{\tau}\left(\mu_{k}+Q_{k}\right) \leq Z_{2}^{k \tau}+\Gamma_{2}^{\tau}\left(\mu_{k}+Q_{k}\right) \\
& Z_{1}^{k \tau}+\Gamma_{1}^{\tau}\left(\mu_{k}+Q_{k}\right) \leq Z_{a}^{k \tau}+\Gamma_{a}^{\tau}\left(\mu_{k}+Q_{k}\right)
\end{aligned}
$$

And the optimality of $p_{2}$ for the empty truck suggests the following:

$$
\begin{aligned}
& Z_{2}^{k \tau}+\Gamma_{2}^{\tau}\left(\mu_{k}+0\right) \leq Z_{1}^{k \tau}+\Gamma_{1}^{\tau}\left(\mu_{k}+0\right) \\
& Z_{2}^{k \tau}+\Gamma_{2}^{\tau}\left(\mu_{k}+0\right) \leq Z_{a}^{k \tau}+\Gamma_{a}^{\tau}\left(\mu_{k}+0\right)
\end{aligned}
$$

Now, to prove the proposed "if and only if" statement a two-way proof must be given:

Part 1 (forward proof): Path $p_{a}$ is a fuel consumption minimising path for truck type $k$ carrying some load $f \in\left[\frac{Z_{a}^{k \tau}-Z_{2}^{k \tau}}{\Gamma_{2}^{\tau}-\Gamma_{a}^{\tau}}-\right.$ $\left.\mu_{k}, \frac{Z_{1}^{k \tau}-Z_{a}^{k \tau}}{\Gamma_{a}^{\tau}-\Gamma_{1}^{\tau}}-\mu_{k}\right] \Rightarrow$ path $p_{a}$ is an eligible path: if we only prove that $\frac{Z_{a}^{k \tau}-Z_{2}^{k \tau}}{\Gamma_{2}^{\tau}-\Gamma_{a}^{\tau}}-\mu_{k} \geq 0$, and $\frac{Z_{1}^{k \tau}-Z_{a}^{k \tau}}{\Gamma_{a}^{\tau}-\Gamma_{1}^{\tau}}-\mu_{k} \leq Q_{k}$, then we have proved $f \in\left[0, Q_{k}\right]$, which then makes the stated assumption per se sufficient for the eligibility of $p_{a}$ (note that $\frac{Z_{a}^{k \tau}-Z_{2}^{k \tau}}{\Gamma_{2}^{\tau}-\Gamma_{a}^{\tau}}-\mu_{k} \leq \frac{Z_{1}^{k \tau}-Z_{a}^{k \tau}}{\Gamma_{a}^{\tau}-\Gamma_{1}^{\tau}}-\mu_{k}$ is already assumed). A proof by contradiction can be used where we assume either $\frac{Z_{a}^{k \tau}-Z_{2}^{k \tau}}{\Gamma_{2}^{\tau}-\Gamma_{a}^{\tau}}-\mu_{k}<0$, or $\frac{Z_{1}^{k \tau}-Z_{a}^{k \tau}}{\Gamma_{a}^{\tau}-\Gamma_{1}^{\tau}}-\mu_{k}>Q_{k}$. If $\frac{Z_{a}^{k \tau}-Z_{2}^{k \tau}}{\Gamma_{2}^{\tau}-\Gamma_{a}^{\tau}}-\mu_{k}<0$, since it is equivalent to $Z_{a}^{k \tau}+\Gamma_{a}^{\tau} \mu_{k}<Z_{2}^{k \tau}+\Gamma_{2}^{\tau} \mu_{k}$, we will have a contradiction with (8). At the same time, if $\frac{Z_{1}^{k \tau}-Z_{a}^{k \tau}}{\Gamma_{a}^{\tau}-\Gamma_{1}^{\tau}}-\mu_{k}>Q_{k}$, since it is equivalent to $Z_{1}^{k \tau}+$ $\Gamma_{1}^{\tau}\left(\mu_{k}+Q_{k}\right)>Z_{a}^{k \tau}+\Gamma_{a}^{\tau}\left(\mu_{k}+Q_{k}\right)$, we will have a contradiction with (6). Therefore, neither $\frac{Z_{a}^{k \tau}-Z_{2}^{k \tau}}{\Gamma_{2}^{\tau}-\Gamma_{a}^{\tau}}-\mu_{k}<0$, nor $\frac{Z_{1}^{k \tau}-Z_{a}^{k \tau}}{\Gamma_{a}^{\tau}-\Gamma_{1}^{\tau}}-\mu_{k}>Q_{k}$, and as $f \in\left[0, Q_{k}\right]$, path $p_{a}$ is a fuel consumption minimising path for truck type $k$ carrying some load $f \in\left[0, Q_{k}\right]$ and is hence an eligible path based on definition.

Part 2 (backward proof): path $p_{a}$ is an eligible path $\Rightarrow$ Path $p_{a}$ is a fuel consumption minimising path for truck type $k$ carrying some load $f \in\left[\frac{Z_{a}^{k \tau}-Z_{2}^{k \tau}}{\Gamma_{2}^{\tau}-\Gamma_{a}^{\tau}}-\mu_{k}, \frac{Z_{1}^{k \tau}-Z_{a}^{k \tau}}{\Gamma_{a}^{\tau}-\Gamma_{1}^{\tau}}-\mu_{k}\right]$ : The eligibility of $p_{a}$ necessitates that both of the following inequalities hold for some $f \in\left[0, Q_{k}\right]$ :

$$
\begin{aligned}
& Z_{a}^{k \tau}+\Gamma_{a}^{\tau}\left(\mu_{k}+f\right) \leq Z_{1}^{k \tau}+\Gamma_{1}^{\tau}\left(\mu_{k}+f\right) \\
& Z_{a}^{k \tau}+\Gamma_{a}^{\tau}\left(\mu_{k}+f\right) \leq Z_{2}^{k \tau}+\Gamma_{2}^{\tau}\left(\mu_{k}+f\right)
\end{aligned}
$$

Since (9) is equivalent to $\frac{Z_{1}^{k \tau}-Z_{a}^{k \tau}}{\Gamma_{a}^{\tau}-\Gamma_{1}^{\tau}}-\mu_{k} \geq f$, and (10) is equivalent to $\frac{Z_{a}^{k \tau}-Z_{2}^{k \tau}}{\Gamma_{2}^{\tau}-\Gamma_{a}^{\tau}}-\mu_{k} \leq f$, we have $\frac{Z_{a}^{k \tau}-Z_{2}^{k \tau}}{\Gamma_{2}^{\tau}-\Gamma_{a}^{\tau}}-\mu_{k} \leq f \leq$ $\frac{Z_{1}^{k \tau}-Z_{a}^{k \tau}}{\Gamma_{a}^{\tau}-\Gamma_{1}^{\tau}}-\mu_{k}$ 
Corollary 1 If for a given departure time $\tau$, path $p_{a}$ is a fuel consumption minimising path for a truck of type $k$ carrying load $f_{a}=\frac{Z_{1}^{k \tau}-Z_{2}^{k \tau}}{\Gamma_{2}^{\tau}-\Gamma_{1}^{\tau}}-\mu_{k}$, then $p_{a}$ is an eligible path.

Proof. Based on Theorem 2 we must prove that $f_{a} \in\left[\frac{Z_{a}^{k \tau}-Z_{2}^{k \tau}}{\Gamma_{2}^{\tau}-\Gamma_{a}^{\tau}}-\mu_{k}, \frac{Z_{1}^{k \tau}-Z_{a}^{k \tau}}{\Gamma_{a}^{\tau}-\Gamma_{1}^{\tau}}-\mu_{k}\right]$; that is, we must prove $\frac{Z_{a}^{k \tau}-Z_{2}^{k \tau}}{\Gamma_{2}^{\tau}-\Gamma_{a}^{\tau}} \leq$ $\frac{Z_{1}^{k \tau}-Z_{2}^{k \tau}}{\Gamma_{2}^{\tau}-\Gamma_{1}^{\tau}} \leq \frac{Z_{1}^{k \tau}-Z_{a}^{k \tau}}{\Gamma_{a}^{\tau}-\Gamma_{1}^{\tau}}$. The optimality of $p_{a}$ for the truck carrying load $f_{a}=\frac{Z_{1}^{k \tau}-Z_{2}^{k \tau}}{\Gamma_{2}^{\tau}-\Gamma_{1}^{\tau}}-\mu_{k}$ yields:

$$
\begin{aligned}
& Z_{a}^{k \tau}+\Gamma_{a}^{\tau}\left(\frac{Z_{1}^{k \tau}-Z_{2}^{k \tau}}{\Gamma_{2}^{\tau}-\Gamma_{1}^{\tau}}\right) \leq Z_{1}^{k \tau}+\Gamma_{1}^{\tau}\left(\frac{Z_{1}^{k \tau}-Z_{2}^{k \tau}}{\Gamma_{2}^{\tau}-\Gamma_{1}^{\tau}}\right) \\
& Z_{a}^{k \tau}+\Gamma_{a}^{\tau}\left(\frac{Z_{1}^{k \tau}-Z_{2}^{k \tau}}{\Gamma_{2}^{\tau}-\Gamma_{1}^{\tau}}\right) \leq Z_{2}^{k \tau}+\Gamma_{2}^{\tau}\left(\frac{Z_{1}^{k \tau}-Z_{2}^{k \tau}}{\Gamma_{2}^{\tau}-\Gamma_{1}^{\tau}}\right)
\end{aligned}
$$

where (11) is equivalent to $\frac{Z_{1}^{k \tau}-Z_{2}^{k \tau}}{\Gamma_{2}^{\tau}-\Gamma_{1}^{\tau}} \leq \frac{Z_{1}^{k \tau}-Z_{a}^{k \tau}}{\Gamma_{a}^{\tau}-\Gamma_{1}^{\tau}}$, and (12) is equivalent to $\frac{Z_{a}^{k \tau}-Z_{2}^{k \tau}}{\Gamma_{2}^{\tau}-\Gamma_{a}^{\tau}} \leq \frac{Z_{1}^{k \tau}-Z_{2}^{k \tau}}{\Gamma_{2}^{\tau}-\Gamma_{1}^{\tau}}$.

In order to understand better the proposed results and the proofs, we further provide some visual presentations in Figure 2. In Figure 2.a, the lines that correspond to the equations of the fuel consumption minimising paths for a full and an empty truck $k$ at time instant $\tau$, are given as $p_{1}$ and $p_{2}$, respectively. Figure 2.b illustrates an ineligible path that can never minimise the truck fuel consumption at any load level within the truck capacity. Figure 2.c, illustrates an eligible path satisfying all conditions set in the Theorem. This figure shows further the condition set in Corollary 1. Finally, Figure 2.d shows two different eligible paths satisfying all conditions. Obviously, no line of an eligible path could be sketched without its eligibility range being within the one defined in Theorem 2.

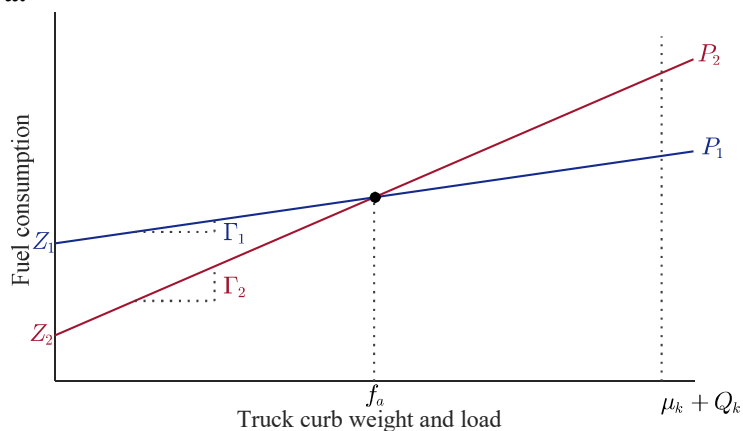

c.

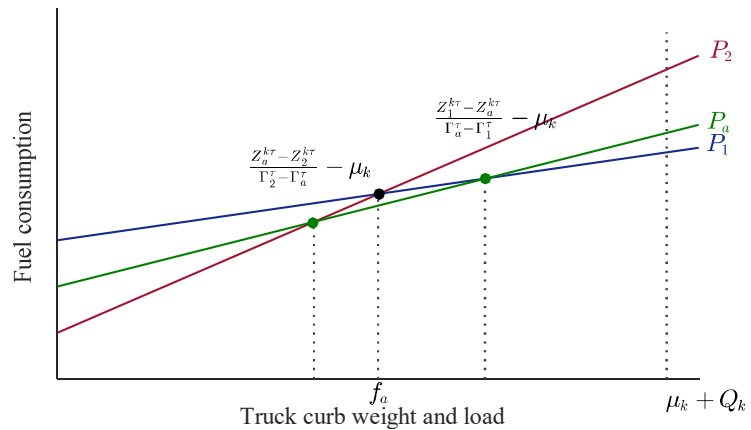

b.

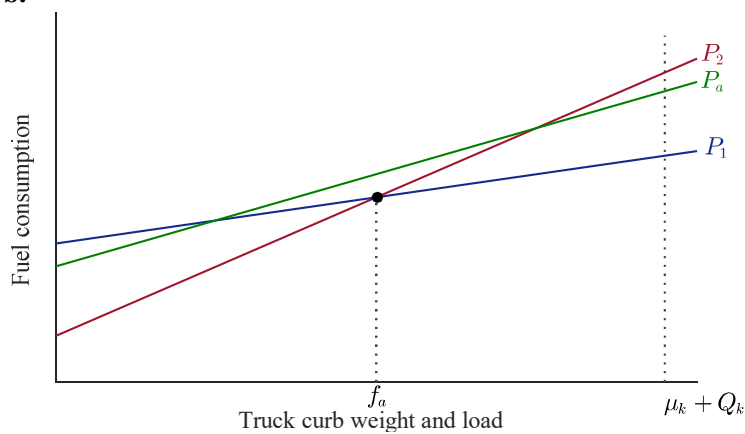

d.

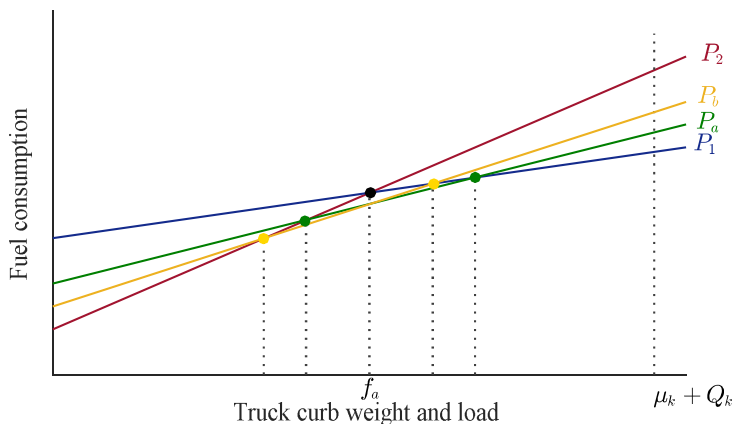

Figure 2 a. Paths $p_{1}$ and $p_{2}$ and their attributes, b. an ineligible path, c. an eligible path, and d. two different eligible paths

In order to use Theorem 2 in the development of the PEP, we need to generalise it to derive efficient progression and exit conditions for the algorithm:

Corollary 2 Assume that for a given departure time $\tau$, paths $p_{i}$ and $p_{j}$ are two eligible paths, such that $p_{i} \not \equiv p_{j}$. Then, if a distinct path $p_{a}$ is the fuel consumption minimising path for load level $f_{a}=\frac{Z_{i}^{k \tau}-Z_{j}^{k \tau}}{\Gamma_{j}^{\tau}-\Gamma_{i}^{\tau}}-\mu_{k}$, it is an eligible path.

Proof. A proof similar to the one used for corollary 1 can be employed. 
With these results, the PEP is given in Algorithm 1. In this algorithm, the core operation is assigned to the function $\left(p_{\mathfrak{p}}, A T T_{\mathfrak{p}}\right):=\operatorname{TDFCMP}(i, j, \tau, k, f)$, that takes as input the origin and destination nodes $(i, j)$, the departure time $(\tau)$, the truck type $(k)$ and the load carried by the truck $(f)$, and outputs the time-dependent fuel consumption minimising path $\left(p_{\mathfrak{p}}\right)$ and its attributes $\left(A T T_{\mathfrak{p}}\right)$, comprising $t_{\mathfrak{p}}^{\tau}, Z_{\mathfrak{p}}^{k \tau}$ and $\Gamma_{\mathfrak{p}}^{\tau}$, under the given settings. In our implementation, TDFCMP is based on a modified extension of the time-dependent shortest-path algorithm of Ziliaskopoulos and Mahmassani (1993).

In the beginning of the algorithm (line 2) the set $\mathcal{R}_{i j}^{k \tau}, \mathcal{A} \mathcal{T} \mathcal{T}_{i j}^{k \tau}, U P$, and $I L$ are initialised as empty sets to retain eligible paths (for departure time $\tau$, vehicle type $k$ ), their attributes, untreated points, and intersecting lines, respectively. In lines 3 and 4 of the algorithm, the fuel consumption minimising paths for the full and the empty truck are respectively found, and then are compared with each other in line 5. If these two paths are the same, then only one of them is retained and the algorithm is terminated (Proposition 1). Otherwise, the algorithm computes and stores a new untreated point and an intersecting lines pair and goes to line 7 . In lines 7 to 15 , every time a new untreated point is pulled out from the front of the $U P$, and until $U P$ is not empty the operations of these lines are repeated.

Assuming that $|\mathfrak{p}|$ is the cardinality of $\mathcal{R}_{i j}^{k \tau}$, this algorithm must make a maximum of $2|\not p|-1$ calls to the TDFCMP function and hence is quite fast (note only that in the case of $|\not p|=1$ two calls are required and not one). Another speeding up feature that is built in the proposed PEP algorithm is due to the use of the information from customers' time-windows and demands. Note that in line 1 of the algorithm instead of $\tau \in T$ the search space can be significantly reduced by using $\tau \in\left[e_{i}+s_{i}, \min \left(l_{i}+s_{i}, l_{j}\right)\right]$, where $e_{i}+s_{i}$ is the earliest possible departure time, and $\min \left(l_{i}+s_{i}, l_{j}\right)$ is the latest possible departure time from the origin node. Moreover, in lines 3 and 4, instead of using $Q_{k}$ and 0 as input to TDFCMP to identify $p_{1}$ and $p_{2}$, respectively, we have used $Q_{k}-q_{i}$, and $q_{j}$. This is because even if upon departure from the depot the truck is fully loaded, its load over the path from $i$ to $j$ cannot exceed $Q_{k}-q_{i}$, and it is not going to be less than the demand of the destination customer, i.e. $q_{j}$. Note that we assume $q_{0}=0$, so this stays consistent when the origin node is the depot.

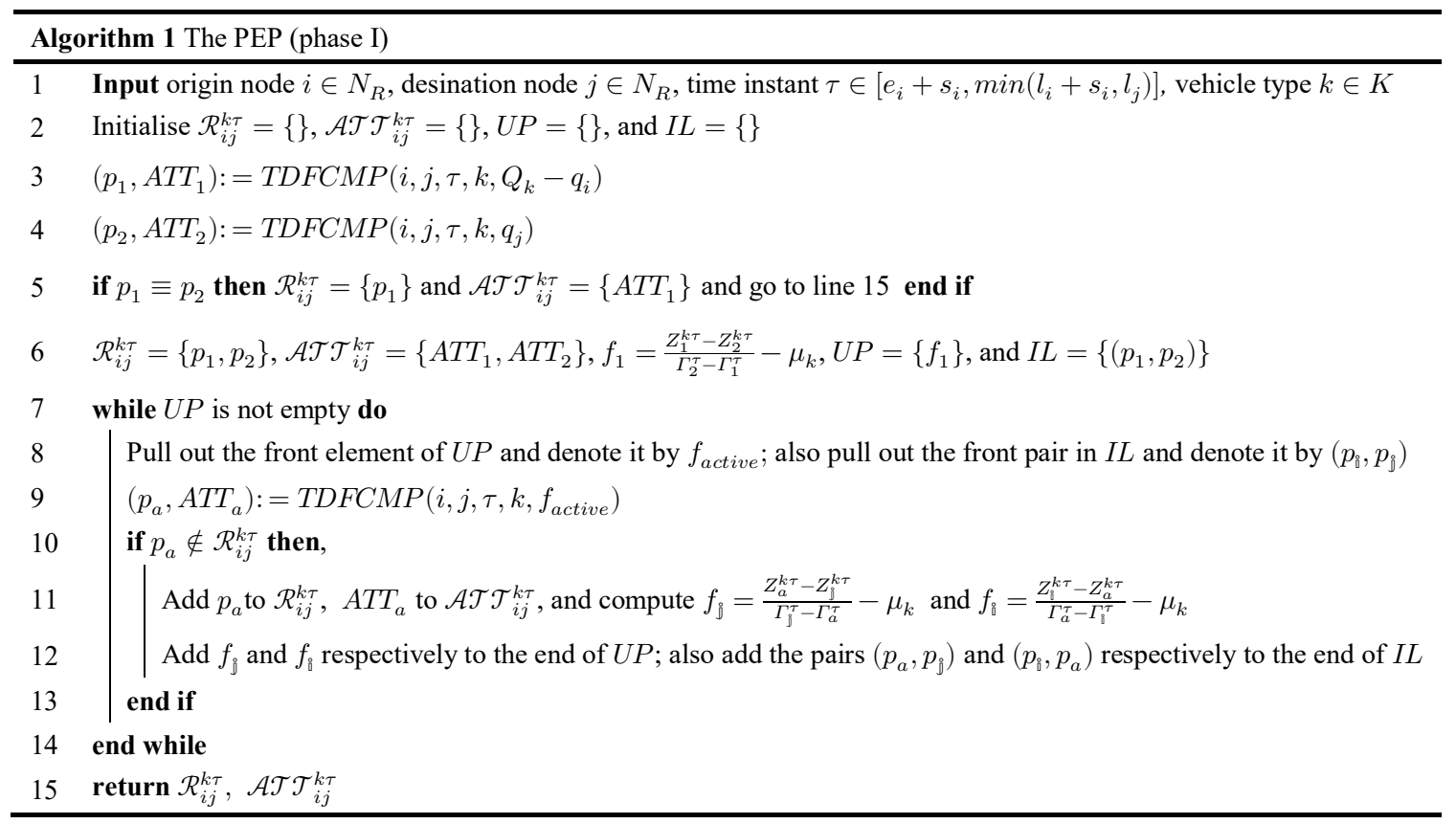

In order to visualise the working of the proposed PEP algorithm, a step-by-step example is illustrated in Figure 3 . In this figure, in the first step, Figure 3.a, $p_{1}$ is found (line 3 of the algorithm), and in the second step, Figure 3.b, $p_{2}$ is found 
(line 4 of the algorithm) and as it is not same as $p_{1}, f_{1}$ is calculated and added to the end of $U P$ (line 5 of the algorithm). The pair $\left(p_{1}, p_{2}\right)$ is also added to the end of $I L$. In the next step, Figure 3.c, $f_{1}$ is extracted from the beginning of $U P$ (line 8 of the algorithm), and the fuel consumption minimising path for the truck at load $f_{1}$ is found (line 9 of the algorithm). Since a different path from $p_{1}$ and $p_{2}$, i.e. path $p_{3}$ is found, it is added to $\mathcal{R}_{i j}^{k \tau}$ and $f_{2}$ and $f_{3}$ are calculated and along with pairs $\left(p_{3}, p_{2}\right)$ and $\left(p_{1}, p_{3}\right)$ are added to the end of $U P$ and $I L$, respectively (line 11 and 12 of the algorithm). In the next step, Figure 3.d, the active point is the first element in $U P$, i.e. $f_{2}$, and a new eligible path is found and the same operations as in the previous step are repeated. After this step, however, as no other new eligible path is found by examining all points in $U P$ (Figure 3.e), the algorithm terminates and returns 4 distinct eligible paths, following a total of 7 calls to the TDFCMP. An interesting outcome of the algorithm is further shown in Figure 3.f, which implies it is possible to know exactly at what load ranges carried by the considered truck at the considered departure time, which path is optimal. In other words, the PEP can return also a piecewise linear function for fuel consumption based on payload.
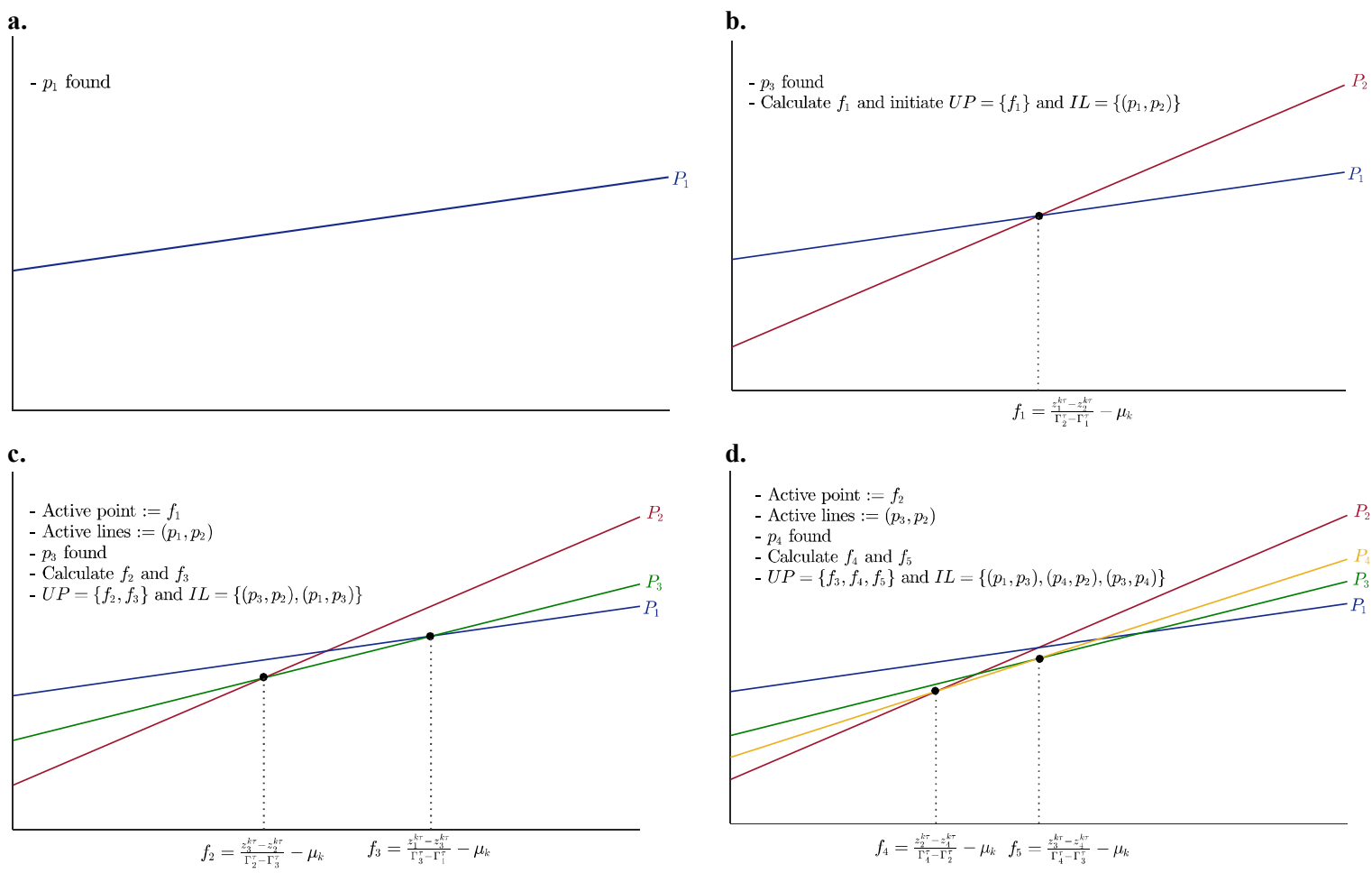

d.

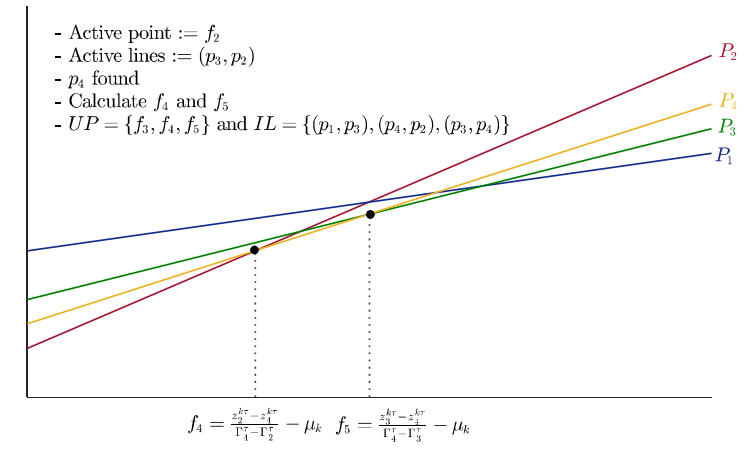

f.
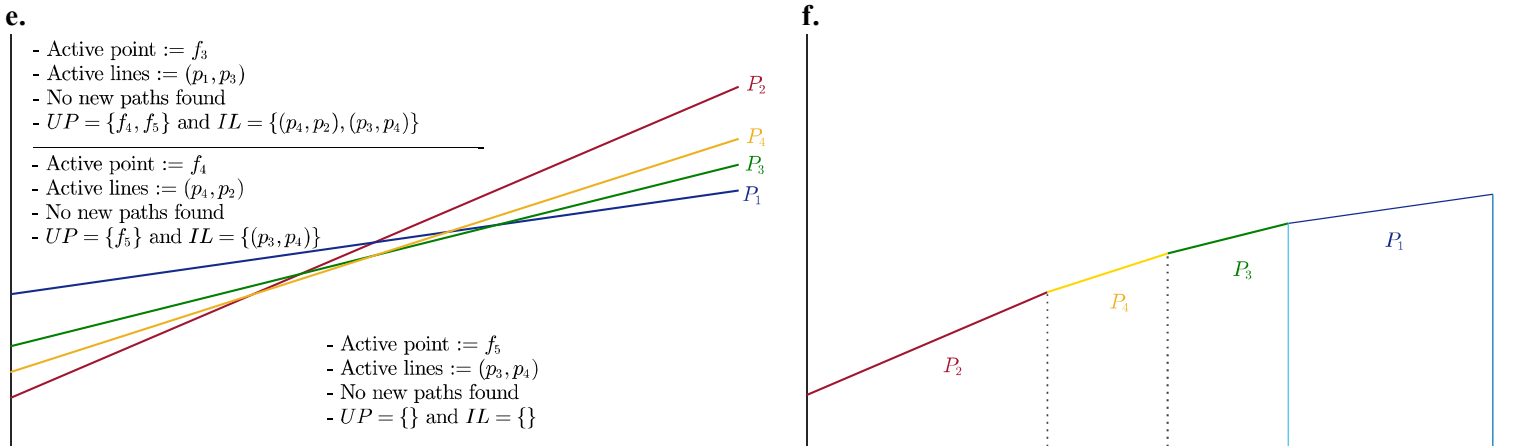

Figure 3 PEP steps

The constructed set $\mathcal{R}_{i j}=\left\{\mathcal{R}_{i j}^{k \tau}: \forall k \in K, \tau \in\left[e_{i}+s_{i}, \min \left(l_{i}+s_{i}, l_{j}\right)\right]\right\}$ after the application of the PEP-phase I, corresponds to the set $\mathcal{D}_{i j}$, and based on Theorem 1, it must be expanded to $\mathcal{E}_{i j}$. However, in most cases no further attempt 
is required for this expansion as the retained set is already equivalent to the set $\mathcal{E}_{i j}$. This is mainly because the fastest path already exists in $\mathcal{R}_{i j}^{\tau}\left(\mathcal{R}_{i j}^{\tau}=\left\{\mathcal{R}_{i j}^{k \tau}: \forall k \in K\right\}\right)$ in most cases (e.g. it is often observed that the fastest path is the fuel consumption minimising path for an empty light duty truck). In any case, for the sake of completeness any necessary further attempt must be identified and carried out in the second phase of the algorithm.

Assume that $p_{\ell}$ is the fastest path in the set $\mathcal{R}_{i j}^{\tau}$ after the application of the PEP phase-I for all vehicle types at time instant $\tau \in T$, and $p_{\mathbb{t}}$ is the globally fastest path at this time instant. Let the ordered set $\mathcal{M}_{i j}^{\tau}=\left\{p_{\mathbb{t}}, p_{\mathbb{t}+1}, \ldots, p_{\mathbb{t}+k}\right\}$ be the set of the $k$ fastest paths at time instant $\tau$, such that $t^{\tau}\left(p_{\mathbb{t}+k}\right)<t^{\tau}\left(p_{\ell}\right)$. Then, none of the paths in the set $\mathcal{M}_{i j}^{\tau}$ are dominated by the paths in $\mathcal{R}_{i j}^{\tau}$ because of their first element in the vector $\left[\boldsymbol{t}^{\tau}\left(p_{i j}\right), \mathfrak{f}_{f}^{k \tau}\left(p_{i j}\right)\right]$ (refer back to Theorem 1 ). However, lower ranked paths in the set $\mathcal{M}_{i j}^{\tau}$ might be dominated by higher ranked paths in this set in terms of fuel consumption; hence, this set could be further refined using the following strong dominance rule:

Proposition 2 At time instant $\tau$, a path $p_{\unrhd}$ in $\mathcal{M}_{i j}^{\tau}$ is not dominated by the higher ranked path $p_{\unrhd}-1$ in $\mathcal{M}_{i j}^{\tau}$ iff $\exists k \in$ $K: \mathfrak{f}_{0}^{k \tau}\left(p_{\triangleright}\right)<\mathfrak{f}_{0}^{k \tau}\left(p_{₫}-1\right)$ and/or $\exists k \in K: \mathfrak{f}_{Q_{k}}^{k \tau}\left(p_{\triangleright}\right)<\mathfrak{f}_{Q_{k}}^{k \tau}\left(p_{₫}-1\right)$.

Proof. The forward statement is obvious and requires no proof; that is, if there is at least one truck $k \in K$ that prefers path $p_{\unrhd}$ over path $p_{\unrhd-1}$ when it is empty and/or when it is fully loaded, then $p_{\S}$ is not dominated by $p_{\S-1}$. Yet, we need to prove the backward statement; i.e.: path $p_{\S}$ is not dominated by path $p_{\S-1} \Rightarrow \exists k \in K: f_{0}^{k \tau}\left(p_{\S}\right)<f_{0}^{k \tau}\left(p_{\S}\right)$ and/or $\exists k \in$ $K: \mathfrak{f}_{Q_{k}}^{k \tau}\left(p_{\triangleright}\right)<\mathfrak{f}_{Q_{k}}^{k \tau}\left(p_{\unrhd}\right)$ : Recall that the domination rule established in Theorem 1 is based on the vector $\left[\boldsymbol{t}^{\tau}\left(p_{\triangleright}\right), \mathfrak{f}_{f}^{k \tau}\left(p_{\triangleright}\right)\right]$, for at least one $\tau \in T, k \in K$, and $f \in\left[0, Q_{k}\right]$. At time instant $\tau$, from the definition of $\mathcal{M}_{i j}^{\tau}$ we know that $t\left(p_{0}^{\tau}\right)<t\left(p_{\square}^{\tau}\right)$; therefore, for path $p_{\S}$ to be not dominated by $p_{\S-1}$, we must have $\mathfrak{f}_{f}^{k \tau}\left(p_{\S}\right)<\mathfrak{f}_{f}^{k \tau}\left(p_{\S-1}\right)$ for at least one $k \in K$, and $f \in$ $\left[0, Q_{k}\right]$, and this is equivalent to (13) below:

$$
Z_{0}^{k \tau}+\Gamma_{\emptyset}^{\tau}\left(\mu_{k}+f\right)<Z_{\emptyset-1}^{k \tau}+\Gamma_{\emptyset-1}^{\tau}\left(\mu_{k}+f\right)
$$

Now, to use a proof by contradiction we assume that path $p_{0}$ is not preferred over path $p_{0-1}$ neither when truck $k$ is empty, nor when it is fully loaded; that is:

$$
\begin{aligned}
& Z_{\AA-1}^{k \tau}+\Gamma_{\llbracket-1}^{\tau} \mu_{k}<Z_{\S}^{k \tau}+\Gamma_{\S}^{\tau} \mu_{k} \\
& Z_{\emptyset-1}^{k \tau}+\Gamma_{\S-1}^{\tau}\left(\mu_{k}+Q_{k}\right)<Z_{\S}^{k \tau}+\Gamma_{\S}^{\tau}\left(\mu_{k}+Q_{k}\right)
\end{aligned}
$$

The combination of (13) and (14) yields that $\Gamma_{\AA}^{\tau}<\Gamma_{\AA-1}^{\tau}$, while the combination of (13) and (15) yields $\Gamma_{\AA}^{\tau}>\Gamma_{\AA-1}^{\tau}$ which is a contradiction.

Hence, the second phase of the PEP is presented in Algorithm 2. Note that in line 3 of the algorithm, a $k$-fastest path algorithm, that takes $t^{\tau}\left(p_{\ell}\right)$ as input, must be used. This algorithm begins by finding the time-dependent fastest path, and loops for the $k$ fastest path where $k=\infty$ or any large number, and breaks out of the loop once the last path found has a travel time greater than or equal to $t^{\tau}\left(p_{\ell}\right)$.

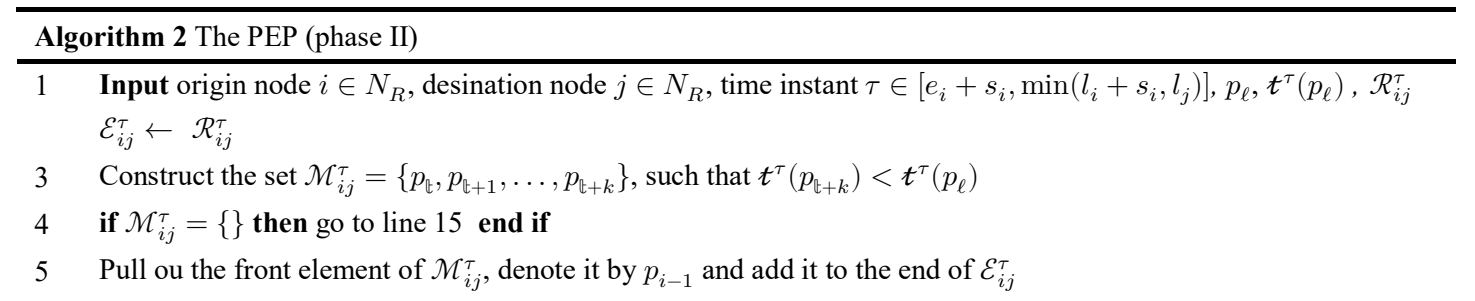




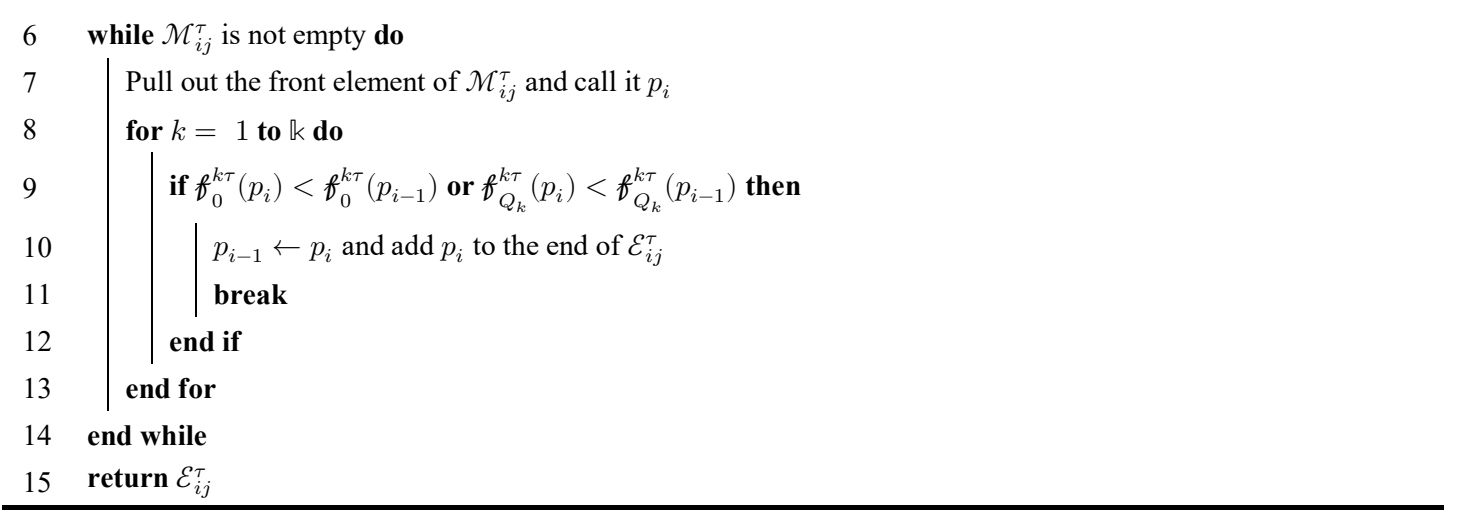

The output of the PEP is a set of retained road-paths between the required nodes with a complete archive of their distance, time-dependent travel times, UTM and RTM attributes in easy-to-access look-up tables, which greatly facilitate the application of any subsequent solution algorithm. However, we still need to generalise the results for the multi-objective case of the SPRP.

Indeed, thanks to the second phase expansion based on paths' travel times, the proposed PEP can be already generalised to the multi-objective case of the SPRP. Let $\hat{G}=(\hat{N}, \hat{A})$ be the resulting multi-graph after the application of the PEP, where $\hat{N}=\left\{N_{0} \cup N_{1}\right\}$, and $\hat{A}$ is the set of retained directed road-paths between the nodes, i.e. $\hat{A}=$ $\{(i, j, p) \mid i, j \in \hat{N}, p \in \mathcal{E}\}$. Then, we propose the following theorem:

Theorem 3 Let $\mathcal{P F}$ be the POS of any instance of the SPRP solved on the reduced graph $\hat{G}$, and $\mathcal{P} \mathcal{F}^{*}$ be the POS of the very SPRP instance solved directly on $G$. Then, $\mathcal{P} \mathcal{F} \equiv \mathcal{P} \mathcal{F}^{*}$.

Proof. We must prove that no Pareto optimal path is discarded from $\hat{G}$ by applying the PEP. Consider a proof by contradiction and suppose that at least for one given departure time instant $\tau$, and a vehicle of type $k$ carrying a load of size $f$, road-path $p_{a} \in G$ between required nodes $i, j \in N_{R}$, which is discarded from $\hat{G}$ by applying the PEP (i.e. $p_{a} \notin \hat{G}$ ), is a Pareto optimal path, and its corresponding non-dominated objective value in the criterion space is $u^{a}=\left(u_{1}^{a}, u_{2}^{a}, u_{3}^{a}\right)$. The fact that $p_{a}$ is excluded from $\hat{G}$ implies that $p_{a} \notin \mathcal{E}_{i j}^{\tau}$ and hence the vector $\left[\boldsymbol{t}^{\tau}\left(p_{a}\right), \mathfrak{f}_{f}^{k \tau}\left(p_{a}\right)\right]$ is a dominated vector. On the other hand, since $u_{2}^{a}=f_{f}^{k \tau}\left(p_{a}\right)$ and $u_{3}^{a}=t^{\tau}\left(p_{a}\right)$, the only way for $u^{a}$ to be a non-dominated vector is to be nondominated based on $u_{1}^{a}$. However, the vehicle hiring cost objective is path-independent.

\subsection{The PEP-based MILP for the SPRP}

A MILP formulation of the SPRP based on the PEP, which is equivalent to a multi-objective, time and load dependent, fleet size and mix PRP, with time windows, flexible departure times, and multi-trips is proposed.

Prior to introducing the decision variables and the model, however, to reduce computational complexity, we need to describe an alternative discretisation of the planning horizon $T$, independently for each road-path $(i, j, p) \in \hat{A}$. As we discussed earlier the time-dependent travel time of a road-link (and hence a road-path) is assumed integer. With this assumption it is probable to have the same travel time at several consecutive departure times. For example, it is possible that if the truck departs the origin of the road-path at any of the consecutive minutes $\{\tau, \tau+1, \tau+2, \ldots, \tau+\ell\} \in T$, the travel times would be equal; i.e. $t_{i j p}^{\tau}=t_{i j p}^{\tau+1}=t_{i j p}^{\tau+2}=\cdots=t_{i j p}^{\tau+\ell}$. Correspondingly, the UTM and the RTM attributes would be the same. Therefore, the whole set of these 'time instants' might be bundled together as a 'time period' $\mathbb{t}$, to 


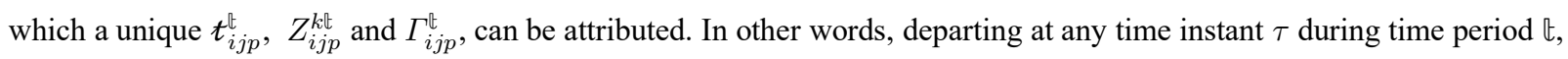
will yield $t_{i j p}^{\sharp}, Z_{i j p}^{k \Downarrow}$ and $\Gamma_{i j p}^{\mathbb{t}}$.

With this explanation, the planning horizon $T$ could be discretised independently for each road-path $(i, j, p) \in \hat{A}$ (using a customised notation $\mathcal{T}_{i j p}$ ), into a number of time periods $h_{i j p}$, proportionate to the changes in the travel time of the path during the planning horizon. Therefore, the discretisation of $\mathcal{T}_{i j p}$ would yield (we drop path indices from $h$ and $\mathcal{T}$ for notational simplicity), $\mathcal{T}=\left\{\left[a_{i j p}^{1}, b_{i j p}^{1}\right],\left[a_{i j p}^{2}, b_{i j p}^{2}\right], \ldots,\left[a_{i j p}^{h}, b_{i j p}^{h}\right]\right\}$, where $a_{i j p}^{1}=e_{0}, b_{i j p}^{h}=l_{0}$, and $a_{i j p}^{\mathbb{L}}$ and $\mathfrak{b}_{i j p}^{\mathbb{L}}$ denote the lower boundary and the upper boundary of time period $\mathbb{E} \in \mathcal{T}$, respectively. Confining this discretisation further by using the information from time-windows, it is possible to impose that $a_{i j p}^{1}=e_{i}+s_{i}$ and $b_{i j p}^{\text {h }}=\min \left(l_{i}+\right.$ $\left.s_{i}, l_{j}\right), \forall(i, j, p) \in \hat{A} \mid i \neq 0$. The following decision variables are then introduced and used by the formulation: (i) the binary variable $x_{i j p}^{k \mathbb{E}}$, which is equal to 1 iff vehicle $k \in K$ departs node $i \in \hat{N}$ during time period $\mathbb{E} \in \mathcal{T}$ to go to node $j \in$ $\hat{N}$, through road-path $(i, j, p) \in \hat{A}$, (ii) the continuous variable $f_{i j p}^{k \mathfrak{t}} \in\left[0, Q_{k}\right]$ which represents the size of load carried by vehicle $k \in K$ over the road-path $(i, j, p) \in \hat{A}$ during time period $\mathbb{t}$, and (iii) the integer variable $y_{i j p}^{k \mathbb{t}}$, which indicates the exact departure time from the origin of path $(i, j, p) \in \hat{A}$ given that it is departed by vehicle $k \in K$ during time period $\mathbb{E}$. Note that $f_{i j p}^{k \downarrow}$ must be time-indexed as it is multiplied by the RTM component in the instantaneous CMEM which is timedependent.

In order to allow vehicles to make multiple trips, assuming that each vehicle is allowed to make a maximum of $r$ trips during the planning horizon, $r$ copies of the set $K$ is added to its end. With this modification, the length of the set $K$ will be $r \vartheta$ and vehicles $\{k+\vartheta, k+2 \vartheta, \ldots, k+r \vartheta\}$ all are the dummy copies of vehicle $k \in K$, but with no assignment cost. It is worth mentioning that the definition of a fixed set of vehicles follows two main reasons and has no contradiction with defining the problem as a fleet size and mix problem; first, the use of the fleet set in the formulation of the problem adds to its generality as it could be simply used for the case of a heterogeneous or homogeneous fixed size fleet as well, and second, it helps multi-trip scheduling. It is clear that if a large enough number of each vehicle type is included in the fleet the problem is a fleet size and mix problem.

The mathematical formulation of the proposed problem is given by (16)-(28).

$$
\begin{aligned}
& z_{1}:=\sum_{(0, i, p) \in \hat{A}} \sum_{k \in K} \sum_{\mathbb{t} \in \mathcal{T}} c_{k} x_{0 i p}^{k \mathfrak{t}} \\
& z_{2}:=\sum_{(i, j, p) \in \hat{A}} \sum_{k \in K} \sum_{\mathbb{t} \in \mathcal{T}} Z_{i j p}^{k \mathfrak{t}} x_{i j p}^{k \mathfrak{t}}+\sum_{(i, j, p) \in \hat{A}} \sum_{\mathbb{t} \in \mathcal{T}} \Gamma_{i j p}^{\mathfrak{t}}\left(\sum_{k \in K} x_{i j p}^{k \mathfrak{k}} \mu_{k}+\sum_{k \in K} f_{i j p}^{k \mathfrak{t}}\right) \\
& z_{3}:=\sum_{(i, 0, p) \in \hat{A}} \sum_{k \in K} \sum_{\mathbb{t} \in \mathcal{T}}\left(y_{i 0 p}^{k \mathfrak{t}}+t_{i 0 p}^{\mathfrak{t}} x_{i 0 p}^{k \mathfrak{k}}\right)-\sum_{(0, i, p) \in \hat{A}} \sum_{k \in K} \sum_{\mathbb{t} \in \mathcal{T}} y_{0 i p}^{k \mathfrak{t}}
\end{aligned}
$$

$\operatorname{Min}\left(z_{1}, z_{2}, z_{3}\right)$

Subject to:

$\sum_{j \in \hat{N}} \sum_{p \in \varepsilon_{i j}} \sum_{k \in K} \sum_{\mathbb{E} \in \mathcal{T}} x_{i j p}^{k \Downarrow t}=1, \quad \forall i \in N_{1}$

$$
\sum_{j \in \hat{N}} \sum_{p \in \mathcal{E}_{i j}} \sum_{\mathbb{t} \in \mathcal{T}} x_{i j p}^{k \mathfrak{t}}-x_{j i p}^{k \Downarrow t}=0, \quad \forall k \in K, i \in \hat{N}
$$

$\sum_{j \in \hat{N}} \sum_{p \in \varepsilon_{i j}} \sum_{k \in K} \sum_{\mathbb{t} \in \mathcal{T}} f_{j i p}^{k \Downarrow}-f_{i j p}^{k \Vdash}=q_{i}, \quad \forall i \in N_{1}$ 


$$
\begin{aligned}
& q_{j} x_{i j p}^{k \mathfrak{\sharp}} \leq f_{i j p}^{k \mathbb{E}} \leq\left(Q_{k}-q_{i}\right) x_{i j p}^{k \mathfrak{t}}, \quad \forall(i, j, p) \in \hat{A}, k \in K, \mathbb{E} \in \mathcal{T} \\
& a_{i j p}^{\sharp} x_{i j p}^{k \Downarrow} \leq y_{i j p}^{k \Downarrow} \leq b_{i j p}^{\mathbb{t}} x_{i j p}^{k \mathfrak{t}}, \quad \forall(i, j, p) \in \hat{A}, k \in K, \mathbb{E} \in \mathcal{T}
\end{aligned}
$$

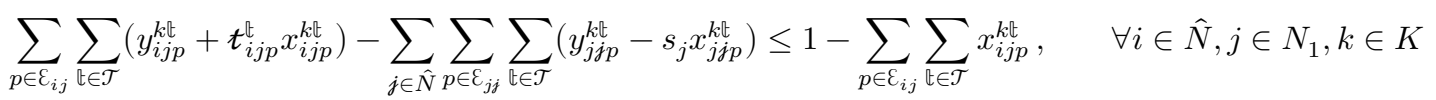

$$
\begin{aligned}
& \sum_{j \in \hat{N}} \sum_{p \in \varepsilon_{i j}} \sum_{k \in K} \sum_{\mathbb{E} \in \mathcal{T}}\left(y_{i j p}^{k \mathbb{t}}-s_{i} x_{i j p}^{k \mathfrak{t}}\right)=\max \left(\sum_{j \in \hat{N}} \sum_{p \in \varepsilon_{i j}} \sum_{k \in K} \sum_{\mathbb{t} \in \mathcal{T}}\left(y_{j i p}^{k \mathfrak{t}}+t_{j i p}^{\mathfrak{t}} x_{j i p}^{k \mathfrak{t}}\right), e_{i}\right), \quad \forall i \in N_{1} \\
& \sum_{j \in \hat{N}} \sum_{p \in \mathcal{E}_{i j}} \sum_{\mathbb{t} \in \mathcal{T}} x_{0 j p}^{k \mathfrak{t}}-x_{0 j p}^{(k+\vartheta) \mathfrak{t}} \geq 0, \quad \forall k \in\{1, \ldots(r-1) \vartheta\} \\
& \sum_{j \in \hat{N}} \sum_{p \in \mathcal{E}_{i j}} \sum_{\mathfrak{t} \in \mathcal{T}} y_{0 j p}^{(k+\vartheta) \mathbb{t}}-\left(y_{j 0 p}^{k \mathfrak{t}}+\left(\boldsymbol{t}_{j 0 p}^{\mathfrak{t}}+s_{0}\right) x_{j 0 p}^{k \mathfrak{k}}\right) \geq M\left(\sum_{j \in \hat{N}} \sum_{p \in \mathcal{E}_{i j}} \sum_{\mathbb{t} \in \mathcal{T}} x_{0 j p}^{(k+\vartheta) \mathfrak{t}}-1\right), \quad \forall k \in\{1, \ldots(\boldsymbol{r}-1) \vartheta\}
\end{aligned}
$$

Expressions (16) to (18) are the objective functions, constraints (20) and (21) are routing constraints, constraints (22) and (23) are capacity and load flow constraints, constraints (24) to (26) are scheduling constraints, and constraints (27) and (28) are multi-trip constraints. The first objective function (16) represents the total hiring cost of the trucks assigned to the routes. Note again that $c_{k}=0, \forall\{k \in K \mid k>\vartheta\}$, so that trucks operating extra rounds of trips are not penalised more than once. The second objective function (17) estimates the total time, load and truck type dependent fuel consumption of the routes; and the third objective (18) represents the total duration of each truck route.

Constraints (20) indicate that each customer must be visited exactly once for delivery. Constraints (21) guarantee that the same vehicle that enters each customer node also exits the node. Constraints (22) model the flow on each road-path. Constraints (23) are used to restrict the total load a vehicle carries by its capacity. Constraints (24) determine the timeperiod during which the origin of a path must be departed. Note that with these constraints $y_{i j p}^{k \Downarrow}$ variables will be a nonnegative integer just for one time period, and zero for all other periods. Constraints (25) tune the time-dependent travel time of each road-path based on the departure time from its origin. Constraints (26) indicate that a customer is departed upon the completion of the service. It is worth noting that through the variable domain definition, we have already implicitly imposed that service takes place within the customers' time windows. Constraints (27) and (28) are multi-trip constraints and together ensure that vehicles could operate another round of trip only if they are back from their first trip and are reloaded for a new one. In (28), $M$ is a sufficiently large number, and without loss of generality could be set equal to $l_{0}+$ $s_{0}$

\section{Generating the full set of the ND points to the SPRP}

It is well-known that Multi-Objective Combinatorial Optimisation (MOCO), dealing with Multi-Objective Integer and Mixed Integer Linear Programming (MOILP/MOMILP) problems, is much more difficult than the Multi-Objective Linear Programming (MOLP), since the feasible set is no longer convex, and unsupported ND points may exist. Hence, even if a complete parameterization on the weight of each objective is attempted, unlike in the MOLP, the ND point set of the problem cannot be fully determined (Alves \& Clímaco, 2007). Some of these methodological difficulties can be easier overcome in the bi-objective case than in the multi-objective one (Alves \& Clímaco, 2007), and this is mainly the reason why most of the solution methodologies in the literature focus on a bi-objective case.

Noticeable developments have been made recently in the area of Mathematical Programming Techniques (MPTs) for the exact solution of tri- and multi-objective integer programming problems, and efficient algorithms have been proposed that can find the full set of the ND points, saving greatly in the total number of IPs required to be solved (Sylva \& Crema, 2004; Özlen \& Azizoglu, 2009; Dhaenens, Lemesre, \& Talbi, 2010; Lokman \& Köksalan, 2013; Özlen, Burton, \& MacRae, 
2014; Boland, Charkhgard, \& Savelsbergh, 2016; Boland, Charkhgard, \& Savelsbergh, 2017). Unfortunately, though, these algorithms cannot be directly applied on the MOMILP problems as infinite number of ND points can lie (e.g. on a line segment) in the continuous parts of the solution space to an MOMILP, and the current state-of-the-art in tackling MOMILPs only allows the consideration of two objectives within a branch-and-bound scheme. However, given the characteristics of the SPRP, the following useful remark allows us to apply directly any successful MPT developed for MOILPs on our problem:

Remark 3 If in an MOMILP continuous variables only appear in at most one of the objective functions, then the given MOMILP has a discrete ND frontier and there is no continuous part in the ND frontier. For such MOMILP, since the ND frontier is discrete, the methods developed for pure MOIPs can be used to find all ND points.

This remark is based on the fact that the projection of the image of a feasible solution to a multi-objective problem in the criterion space with continuous variables in only one of the objectives, is a point, and no line segment can exist along the Pareto frontier (assume a bi-objective problem for visualisation). Therefore, based on this remark, since in the case of the SPRP continuous variables only appear in the objective function related to the fuel consumption minimisation, efficient MPTs for MOILPs are applicable.

We have identified the Quadrant Shrinking Method (QSM) (Boland et al., 2017) as one of the best state-of-the-art MPTs for the exact solution to tri-objective integer programming problems. This MPT needs to solve a maximum of $3\left|y_{\mathbb{N}}\right|+1$ IPs (where $y_{\mathbb{N}}$ is the set of non-dominated points) for the generation of the full set of the ND vectors, and is relatively easy to implement.

For the sake of brevity, an exposition of the QSM method is avoided here and the reader is referred to Boland et al. (2017) for an introduction. To solve the SPRP for the identification of the full set of ND points, we use the PEP-based MILP for the SPRP as the core optimisation problem inside QSM. For returning a ND vector in each iteration of the QSM, two (M)IPs must be solved that correspond to (i) an IP which is a single-objective problem in the third objective of the SPRP, i.e. the total travel time of the routes, and (ii) a MIP which is an aggregation of all the three objectives of the SPRP. This hybridisation turns out to be very efficient, and as will be reported in the computational experiments section of the paper, we are able to find the POS of all instances considered over a reasonable computational time despite the difficulty of the problem.

\section{Construction of realistic spatiotemporal driving cycles}

As discussed earlier, with the current technological advancements in Intelligent Transportation Systems (ITSs) and GPSs it is possible to collect data on fine-grained speeds variations over any given road-link in the road network at different times of a given day using probe vehicles. However, these data are usually unavailable at the planning stage. On the other hand, it has been shown that lack of such data, especially the instantaneous A/D data, can cause inaccuracy in estimating fuel consumption and hence might lead to unreliable and misleading routing decisions (Turkensteen, 2017).

The only relevant study in the area of EMVRPs trying to take this situation into account is a recent study by Kancharla and Ramadurai (2018) who propose to incorporate driving cycles into the estimation of fuel consumption in a timeindependent routing context. The authors collect $450 \mathrm{~km}$ (144 $\mathrm{h}$ of driving) GPS data in the city of Chennai, India, and then for each arc in their test graphs randomly combine the collected micro-trips through an iterative process until the distance of the intended arc is covered. Despite the effort that is put in collecting these data, a major shortcoming of their proposed approach lies in the fact that the spatial and temporal characteristics of the road-links in the graph are completely ignored. 
The location of a road-link in the roadway graph and the time of the day the given road-link is traversed have a fundamental impact on the shape of the speed-time profile and the frequency of vehicles A/Ds.

The spatiotemporal characteristics of a road-link, however, are very well reflected in the macroscopic time-dependent speed data, which are widely available for decision making and could be efficiently used for constructing reliable synthetic driving cycles. Developing microscopic traffic data from macroscopic traffic data based on reconstructed synthetic vehicle trajectories is not something new and is a well-known stream of research in transportation engineering (Zegeye, De Schutter, Hellendoorn, Breunesse, \& Hegyi, 2013; Wang, Daamen, Hoogendoorn, \& Van Arem, 2011; Silvas, Hereijgers, Peng, Hofman, \& Steinbuch, 2016). However, in this paper we adopt a completely different operational research approach and propose a simple but reliable method for the generation of synthetic spatiotemporal driving cycles with using only the road-link distance and the time-dependent average speed as input, and with no parameter tuning. The proposed approach is validated against an extensive library of real-world driving cycles and the results are presented in the 'computational results' section of the paper.

Our proposed approach builds on a model for the generation of worst-case driving cycles, which is then simply weakened with slight parameter relaxation to lead to realistic cycles. Let $d_{i j}$ be the distance of a given road-link $(i, j) \in A$, and $t_{i j}^{t}$ be the time-dependent travel time of the given road-link during time period $\mathbb{E} \in \mathcal{T}$, deduced from the macroscopic traffic speed data using expression (3) (note that it is not necessary to generate the cycles for every time instant of the planning horizon; instead, in line with the arguments in section 4.1, we can generate cycles in the 'time period' level). Also, let $\mathcal{A}_{\text {max }}$ and $\mathcal{D}_{\text {max }}$ respectively denote the maximum possible acceleration and deceleration rates for a truck. Finally, suppose the maximum possible speed in the network is $v_{U}$. Then, the worst-case second-by-second $\mathrm{A} / \mathrm{D}$ rates $\left(a_{t}\right)$ for the given road link during time period $\mathbb{t}$ could be constructed by determining speed levels of every second $\left(v_{t}\right)$ (where $a_{t}=$ $v_{t}-v_{t-1}$ ) using the following nonlinear programming model:

$$
\operatorname{Max} \sum_{t=a_{i j}^{\sharp}+1}^{a_{i j}^{\sharp}+t_{i j}^{\natural}}\left|v_{t}-v_{t-1}\right|
$$

Subject to:

$$
\begin{aligned}
& \sum_{t=a_{i j}^{\sharp}}^{a_{i j}^{\sharp}+t_{i j}^{\sharp}} v_{t}=d_{i j}, \\
& -\mathcal{D}_{\text {max }} \leq v_{t}-v_{t-1} \leq \mathcal{A}_{\text {max }}, \quad \forall t \in\left\{a_{i j}^{\mathbb{t}}+1, a_{i j}^{\mathbb{t}}+2, \ldots, a_{i j}^{\mathbb{t}}+t_{i j}^{\mathbb{t}}\right\} \\
& v_{a_{i j}^{\sharp}}=v_{a_{i j}^{\sharp}+t_{i j}^{\sharp}}=0, \\
& 0 \leq v_{t} \leq v_{U}, \quad \forall t \in\left\{a_{i j}^{\mathbb{t}}, a_{i j}^{\mathbb{t}}+1, \ldots, a_{i j}^{\mathbb{t}}+t_{i j}^{\mathbb{t}}\right\}
\end{aligned}
$$

The nonlinear objective function (29) maximises the positive difference between speed levels of every two consecutive seconds of the cycle, and hence the A/D rates. Constraint (30) tunes the instantaneous speeds in a way that the cycle is completed within the estimated time-dependent travel time of the given road link. Constraints (31) ensure that the $\mathrm{A} / \mathrm{D}$ rates do not violate the maximum possible A/D rate of the truck. Constraint (32) indicate that the truck accelerates from idle (departing node $i$ ) and comes to a full stop at the end of the cycle (arriving at node $j$ ). Note that this is based on the assumption that in an urban road network trucks are usually forced to reduce their speed significantly or come to a full stop at network junctions (e.g. at a cross road traffic light or a turning point). Finally, constraints (33) determine the range of speed values. 
In order to linearize (29)-(33), we define two new non-negative continuous decision variables $A C C_{t}, D E C_{t}, \forall t \in$ $\left\{a_{i j}^{\mathfrak{t}}+1, a_{i j}^{\mathfrak{t}}+2, \ldots, a_{i j}^{\mathfrak{t}}+t_{i j}^{\mathfrak{t}}\right\}$ which indicate the acceleration rates and the deceleration rates during second $t-1$ until $t$, respectively; and a new binary decision variable $x_{t} \in\{0,1\}, \forall t \in\left\{a_{i j}^{\mathbb{t}}+1, a_{i j}^{\mathbb{t}}+2, \ldots, a_{i j}^{\mathbb{t}}+t_{i j}^{\mathbb{t}}\right\}$, which is 1 iff vehicle accelerates during second $t-1$ until $t$, and 0 otherwise. Then, the following MILP which is called the Driving Cycle $\left(D C_{i j}^{\mathfrak{t}}\right)$ model hereafter can be developed:

$$
D C_{i j}^{\sharp}: \operatorname{Max} \sum_{t=a_{i j}^{\sharp}+1}^{a_{i j}^{\sharp}+t_{i j}^{\sharp}} A C C_{t}+D E C_{t}
$$

Subject to:

$$
\begin{aligned}
& v_{t}-v_{t-1}=A C C_{t}-D E C_{t}, \quad \forall t \in\left\{a_{i j}^{\mathbb{t}}+1, a_{i j}^{\mathbb{t}}+2, \ldots, a_{i j}^{\mathbb{t}}+t_{i j}^{\mathbb{t}}\right\} \\
& 0 \leq A C C_{t} \leq x_{t} \mathcal{A}_{\text {max }}, \quad \forall t \in\left\{a_{i j}^{\mathfrak{t}}+1, a_{i j}^{\mathbb{t}}+2, \ldots, a_{i j}^{\mathbb{t}}+t_{i j}^{\mathbb{t}}\right\} \\
& 0 \leq D E C_{t} \leq\left(1-x_{t}\right) \mathcal{D}_{\text {max }}, \quad \forall t \in\left\{a_{i j}^{\mathbb{t}}+1, a_{i j}^{\mathbb{t}}+2, \ldots, a_{i j}^{\mathbb{t}}+t_{i j}^{\mathbb{t}}\right\} \\
& \text { and (30), (32) and (33). }
\end{aligned}
$$

While one can use this model in the development of the robust extension of the SPRP, as we will show later in the computational results section of the paper, very realistic cycles could be generated from the same model only by simply using empirical 'mean' acceleration and deceleration rates $\left(\mathcal{A}_{\text {avg }}\right.$ and $\left.\mathcal{D}_{\text {avg }}\right)$ in the model instead of $\mathcal{A}_{\text {max }}$ and $\mathcal{D}_{\text {max }}$. In this paper, for these parameters we use the reported results by Bokare and Maurya (2017) from their study on the A/D behaviour of various vehicle types including trucks. Based on their results while $\mathcal{A}_{\text {max }} \cong 1 \mathrm{~m} / \mathrm{s}^{2}$, and $\mathcal{D}_{\text {max }} \cong 0.88 \mathrm{~m} / \mathrm{s}^{2}$, the mean acceleration rate of a truck is around $0.3 \mathrm{~m} / \mathrm{s}^{2}$ and the mean deceleration rate is around $0.5 \mathrm{~m} / \mathrm{s}^{2}$. In Figure 4 .a, as a real-world driving cycle, we are illustrating the EPA ${ }^{1}$ heavy duty urban driving schedule which covers a distance of around $8935 \mathrm{~m}$ in $1060 \mathrm{~s}$, with an estimated fuel consumption of 2.55 litres for an empty light-duty truck. Using $D C_{i j}^{\sharp}$ the worst-case driving cycle is generated and illustrated in Figure 4.b with a fuel consumption of 3.22 litres, and the cycle based on the mean A/Ds with a very close amount of fuel consumption to the original cycle, i.e. 2.60 litres, is shown in Figure 4.c.
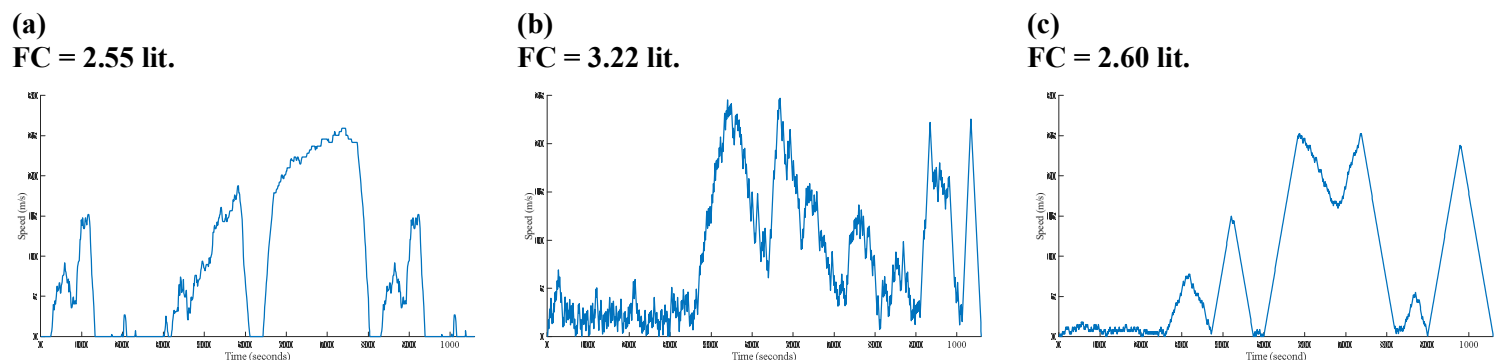

Figure 4 Example synthetic driving cycles constructed from the proposed MILP model

Using the proposed model, if historical microscopic data are unavailable at the planning stage, it is possible to generate reliable driving cycles without collecting field data, and estimate fuel consumption more accurately than using the average-speed CMEM. To generate the required cycles for all network links at all time periods, the proposed model must be used iteratively. An intuitive iterative algorithm for this purpose is presented in Appendix C. In the next section, we report the computational cost of generating the network-wide driving cycles in the case of each instance using this algorithm.

\footnotetext{
${ }^{1}$ https://www.epa.gov/sites/production/files/2015-10/huddscol.txt
} 
As a wrapper of all modules and processes introduced in the paper, in the flow chart in Figure 5, we present the flow of the operations that are carried out on the original roadway graph until the full set of the ND points to an instance of the SPRP are identified. In this flow chart, we are indicating the section of the paper that is relevant to each of the four main modules introduced.

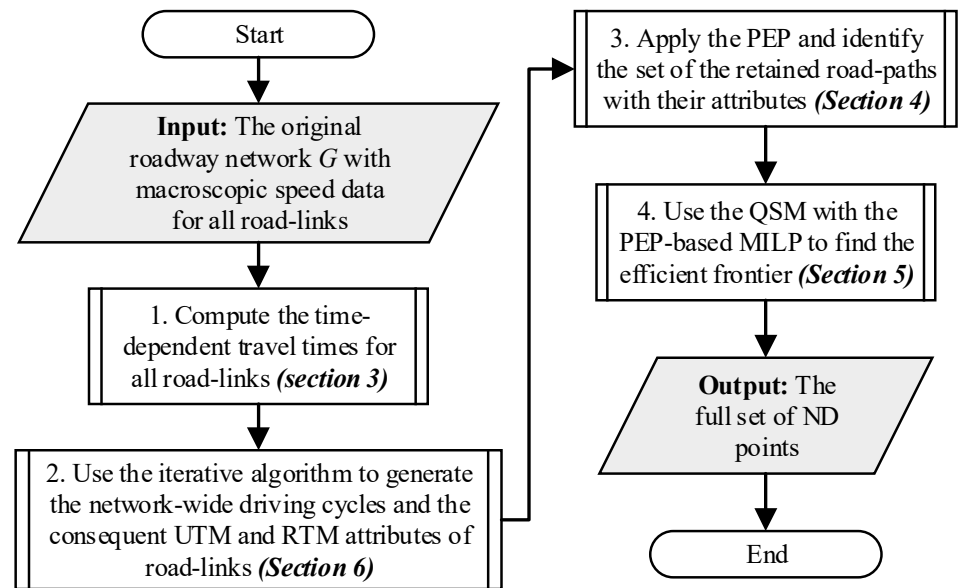

Figure 5 The flow of modules introduced in the paper until finding the full set of the ND points

\section{Computational results}

A set of time-dependent roadway graphs with 100 nodes are created as the test bed of the proposed PEP-based MILP. For these instances, the raw graphs are generated using the procedure proposed by Letchford et al. (2013) that leads to graphs which resemble real-life road networks. To generate time-dependent travel times for the arcs, a planning horizon of 480 minutes is assumed, and traffic condition is supposed to follow a non-congested/congested/non-congested/congested pattern. The two congested periods represent the morning and evening rush hours with speed values generated randomly within the range 15-40 $\mathrm{km} / \mathrm{h}$. Non-congested speeds, on the other hand, are determined randomly within the range 40-70 $\mathrm{km} / \mathrm{h}$. Within this pattern, random speed observations are generated independently for each arc in 15-minute increments (i.e. 32 speed observations per road-link) such that speed levels change from one period to the next smoothly. Following this, the time-dependent travel times of arcs are computed per minute using the proposed closed form formula (3) and rounded up to the nearest integer. For all arcs, driving cycles are generated using $D C_{i j}^{\mathbb{t}}$ MILP for each time period, and the resulting UTM and RTM attributes of the arcs are computed and stored.

Across the generated road networks, we have randomly selected 10 customers to be served by a central depot. For multi-objective experiments, to be able to generate the full set of the ND points on the efficient frontier within a reasonable time, we have considered 5 customers. It is worth mentioning that while the dimension of the proposed instances compares well with other existing papers with an exact approach given the extra complications from multi-trips and flexible paths, it is not an intention of this paper to solve large size test instances, and it has been the task of our follow up paper on the development of multi-objective optimisation heuristics for the SPRP. Instead, here we are more interested in observing the performance of the PEP, the contributions of the multi-trips, the trade-offs on the Pareto front of the SPRP and presenting a benchmark for examining the performance of future heuristics for the problem.

In all test instances, service times and the reloading time at the depot for vehicles executing an extra round of trip are assumed 20 minutes. Feasible time-windows and demands are induced for the customers using a procedure based on a nearest neighbour algorithm where a heavy-duty truck is dispatched to visit the nearest customer in each iteration of the algorithm, until capacity or time constraints are violated. Customers' demands are drawn randomly from the discrete uniform distribution on the interval $[1000 \mathrm{~kg}, 15000 \mathrm{~kg}$ ], and relatively wide time-windows covering up to $40 \%$ of the 
planning horizon are generated around the arrival time of the dispatched trucks. For all instances, the fleet is supposed to be composed of two light-duty, two medium-duty, and two heavy-duty trucks, and all trucks are allowed to execute a maximum of two rounds of trips during the planning horizon. All the test instances developed in this paper along with the reported solutions in this section are available at: https://dx.doi.org/10.17635/lancaster/researchdata/266, or could be requested from the authors via email.

All the experiments were performed on a computer with Intel Core ${ }^{\mathrm{TM}}$ i5 $3.20 \mathrm{GHz}$ processor with 8 GB RAM. The branch-and-bound solver of CPLEX ${ }^{\mathrm{TM}}$ 12.6.3 was used as the exact solver, and except for the PEP and the travel time calculation algorithms that were coded in MATLAB ${ }^{\mathrm{TM}}$, all other algorithms were modelled as OPL scripts in preprocessing, post-processing and model flow control on top of the OPL models of the core MILPs. No global time limit was used to allow the solver to generate the full set of the ND points for benchmarking purposes.

\subsection{Performance of the PEP}

To demonstrate the efficiency of the proposed PEP and the MILP based on it in addressing the SPRP, all test instances with 10 customers are solved to optimality for the fuel consumption minimisation objective using the PEP-based MILP, and solutions are compared with the solutions from a TDSP-based MILP, where a multi-graph based on the full set of the TDSPs between required nodes is used. The results of this comparison are presented in Table 2. In this table, the total number of arcs in the original networks, and in the reduced networks after applying the PEP and the TDSP are shown, along with the litres of fuel consumed (FC) by the optimal solutions returned by each of the formulations, and the runtime (in minutes). One column is also devoted to reporting the percentage optimality gap of the TDSP-based formulation from the optimal solution returned by the PEP-based formulation. Table 2 shows that for all the instances considered, the solution based on the TDSP is suboptimal. The table also indicates that TDSPs on average can only represent less than $44 \%$ of the eligible road-paths in the graph. Observe that the runtime columns of the table suggest that despite the difficulty of the problem, the proposed MILP formulation can find the optimal solutions in a reasonable runtime with an average of less than 10 minutes.

Table 2

Optimal fuel consumption yielded by the PEP-based and the TDSP-based formulations

\begin{tabular}{|c|c|c|c|c|c|c|c|c|}
\hline \multirow{2}{*}{$\begin{array}{l}\text { Instance } \\
\#\end{array}$} & \multirow{2}{*}{$\begin{array}{l}\text { Network } \\
\text { Arcs }\end{array}$} & \multicolumn{3}{|c|}{ PEP-based MILP } & \multicolumn{4}{|c|}{ TDSP-based MILP } \\
\hline & & Arcs & $\mathrm{FC}$ & $\begin{array}{l}\text { Runtime } \\
\text { (min) }\end{array}$ & Arcs & $\mathrm{FC}$ & $\begin{array}{l}\text { Runtime } \\
\text { (min) }\end{array}$ & Gap \\
\hline 1 & 344 & 149 & 54.24 & 11.03 & 109 & 55.56 & 29.72 & $2.43 \%$ \\
\hline 2 & 370 & 270 & 79.32 & 1.26 & 82 & 86.43 & 0.23 & $8.96 \%$ \\
\hline 3 & 358 & 195 & 55.36 & 18.03 & 127 & 57.27 & 4.68 & $3.46 \%$ \\
\hline 4 & 348 & 187 & 77.06 & 1.94 & 96 & 84.64 & 4.06 & $9.83 \%$ \\
\hline 5 & 362 & 259 & 65.85 & 5.26 & 112 & 69.97 & 3.28 & $6.26 \%$ \\
\hline 6 & 366 & 182 & 68.30 & 1.02 & 92 & 74.21 & 0.26 & $8.65 \%$ \\
\hline 7 & 354 & 247 & 59.09 & 32.20 & 152 & 63.38 & 80.98 & $7.26 \%$ \\
\hline 8 & 358 & 218 & 71.56 & 0.76 & 108 & 79.02 & 0.92 & $10.42 \%$ \\
\hline 9 & 374 & 190 & 82.15 & 3.44 & 131 & 84.80 & 3.45 & $3.22 \%$ \\
\hline 10 & 350 & 208 & 82.95 & 0.82 & 117 & 90.14 & 0.79 & $8.67 \%$ \\
\hline 11 & 358 & 172 & 56.49 & 4.65 & 119 & 58.99 & 10.72 & $4.42 \%$ \\
\hline 12 & 360 & 157 & 81.25 & 0.90 & 90 & 87.60 & 0.60 & $7.82 \%$ \\
\hline 13 & 362 & 180 & 67.39 & 1.39 & 96 & 68.86 & 0.50 & $2.18 \%$ \\
\hline 14 & 358 & 214 & 79.84 & 1.62 & 121 & 85.51 & 1.35 & $7.11 \%$ \\
\hline 15 & 360 & 247 & 49.12 & 15.86 & 145 & 51.15 & 12.62 & $4.13 \%$ \\
\hline 16 & 348 & 149 & 60.28 & 0.28 & 99 & 65.75 & 0.86 & $9.08 \%$ \\
\hline 17 & 412 & 210 & 65.48 & 3.76 & 102 & 69.25 & 1.47 & $5.76 \%$ \\
\hline 18 & 348 & 158 & 78.31 & 1.71 & 89 & 86.21 & 1.36 & $10.09 \%$ \\
\hline 19 & 426 & 238 & 51.58 & 5.21 & 102 & 58.01 & 6.77 & $12.48 \%$ \\
\hline 20 & 352 & 167 & 71.20 & 0.23 & 85 & 77.27 & 0.22 & $8.53 \%$ \\
\hline 21 & 346 & 248 & 77.16 & 3.96 & 99 & 82.73 & 0.54 & $7.22 \%$ \\
\hline
\end{tabular}




\begin{tabular}{lllllllll}
22 & 370 & 211 & 76.04 & 0.59 & 106 & 81.58 & 0.71 & $7.27 \%$ \\
23 & 350 & 200 & 77.47 & 1.93 & 103 & 85.20 & 0.49 & $9.96 \%$ \\
24 & 368 & 221 & 77.80 & 11.52 & 103 & 83.87 & 1.43 & $7.80 \%$ \\
25 & 362 & 182 & 78.69 & 2.03 & 83 & 87.70 & 1.89 & $11.45 \%$ \\
\hline Avg. & 362.56 & 202.36 & 69.76 & 5.26 & 106.72 & 75.00 & 3.33 & $7.52 \%$ \\
\hline
\end{tabular}

A more in-depth analysis of the performance of the PEP and the three other approximate algorithms on a real road network based on the Chicago's arterial streets is provided in Appendix B.

\subsection{Fleet size and mix, and the effect of using multiple-trips}

The fleet size and mix of the optimal solutions to instances with 10 customers in case of the fuel consumption minimisation is analysed and presented in Table 3. In this table, the column ' $\mathrm{C}$ ' indicates the cost of hiring the trucks in the solution, and the column ' $R$ ' denotes the number of routes in the solution. Number of each vehicle type light-duty (L), medium-duty (M) and heavy-duty $(\mathrm{H})$ employed by the solution is presented under the heading 'NU'. Average percentage of capacity of each truck type used when departing the depot is shown by 'CU', and the number of each truck type employed for multiple trips is given under the heading 'MT'. Since only medium-duty trucks are selected by all solutions for multi-trips, the average percentage of their capacity used for the second round of delivery is shown in column 'CUM'. Finally, the last two columns of the table denote the percentage savings in fuel consumption and cost due to the use of multi-trips. To measure the savings, we have prohibited multi-trips from all instances and then re-optimised the problems for fuel consumption minimisation and calculated the deviation. Note that in case of instance $\# 9$ the problem is infeasible if multi-trips are not allowed.

Table 3

Fleet size and mix, average capacity use and the use of the multiple-trips in the optimal fuel consuming solutions

\begin{tabular}{|c|c|c|c|c|c|c|c|c|c|c|c|c|c|c|}
\hline \multirow{2}{*}{ Inst. } & \multirow{2}{*}{$\mathrm{C}$} & \multirow{2}{*}{$\mathrm{R}$} & \multicolumn{3}{|l|}{ NU } & \multicolumn{3}{|l|}{$\mathrm{CU}$} & \multicolumn{3}{|l|}{ MT } & \multirow{2}{*}{$\begin{array}{l}\text { CUM } \\
M\end{array}$} & \multicolumn{2}{|c|}{ MT savings } \\
\hline & & & $\mathrm{L}$ & $\mathrm{M}$ & $\mathrm{H}$ & $\mathrm{L}$ & $M$ & $\mathrm{H}$ & $\mathrm{L}$ & $\mathrm{M}$ & $\mathrm{H}$ & & Fuel & $\cos t$ \\
\hline 1 & 162 & 5 & 1 & 2 & 0 & $79 \%$ & $91 \%$ & - & 0 & 2 & 0 & $68 \%$ & $2 \%$ & $84 \%$ \\
\hline 2 & 214 & 4 & 0 & 2 & 1 & - & $74 \%$ & $94 \%$ & 0 & 1 & 0 & $82 \%$ & $2 \%$ & $16 \%$ \\
\hline 3 & 120 & 4 & 0 & 2 & 0 & - & $74 \%$ & - & 0 & 2 & 0 & $77 \%$ & $9 \%$ & $78 \%$ \\
\hline 4 & 308 & 4 & 0 & 2 & 2 & - & $60 \%$ & $82 \%$ & 0 & 0 & 0 & - & $0 \%$ & $0 \%$ \\
\hline 5 & 204 & 5 & 2 & 2 & 0 & $98 \%$ & $63 \%$ & - & 0 & 1 & 0 & $97 \%$ & $4 \%$ & $46 \%$ \\
\hline 6 & 214 & 4 & 0 & 2 & 1 & - & $76 \%$ & $89 \%$ & 0 & 1 & 0 & $64 \%$ & $3 \%$ & $44 \%$ \\
\hline 7 & 120 & 4 & 0 & 2 & 0 & - & $77 \%$ & - & 0 & 2 & 0 & $79 \%$ & $2 \%$ & $148 \%$ \\
\hline 8 & 214 & 4 & 0 & 2 & 1 & - & $53 \%$ & $75 \%$ & 0 & 1 & 0 & $80 \%$ & $0 \%$ & $39 \%$ \\
\hline 9 & 392 & 8 & 2 & 2 & 2 & $92 \%$ & $82 \%$ & $73 \%$ & 0 & 2 & 0 & $77 \%$ & - & - \\
\hline 10 & 214 & 5 & 0 & 2 & 1 & - & $54 \%$ & $86 \%$ & 0 & 2 & 0 & $85 \%$ & $3 \%$ & $64 \%$ \\
\hline 11 & 204 & 5 & 2 & 2 & 0 & $73 \%$ & $81 \%$ & - & 0 & 1 & 0 & $94 \%$ & $3 \%$ & $0 \%$ \\
\hline 12 & 214 & 5 & 0 & 2 & 1 & - & $68 \%$ & $94 \%$ & 0 & 2 & 0 & $75 \%$ & $2 \%$ & $44 \%$ \\
\hline 13 & 298 & 6 & 2 & 2 & 1 & $68 \%$ & $85 \%$ & $87 \%$ & 0 & 1 & 0 & $67 \%$ & $2 \%$ & $32 \%$ \\
\hline 14 & 298 & 6 & 2 & 2 & 1 & $84 \%$ & $51 \%$ & $51 \%$ & 0 & 1 & 0 & $80 \%$ & $0 \%$ & $0 \%$ \\
\hline 15 & 162 & 4 & 1 & 2 & 0 & $73 \%$ & $67 \%$ & - & 0 & 1 & 0 & $80 \%$ & $10 \%$ & $58 \%$ \\
\hline 16 & 214 & 5 & 0 & 2 & 1 & - & $83 \%$ & $58 \%$ & 0 & 2 & 0 & $86 \%$ & $5 \%$ & $44 \%$ \\
\hline 17 & 214 & 4 & 0 & 2 & 1 & - & $95 \%$ & $97 \%$ & 0 & 1 & 0 & $74 \%$ & $4 \%$ & $44 \%$ \\
\hline 18 & 214 & 5 & 0 & 2 & 1 & - & $83 \%$ & $69 \%$ & 0 & 2 & 0 & $75 \%$ & $2 \%$ & $44 \%$ \\
\hline 19 & 204 & 5 & 2 & 2 & 0 & $79 \%$ & $59 \%$ & - & 0 & 1 & 0 & $78 \%$ & $0 \%$ & $46 \%$ \\
\hline 20 & 214 & 4 & 0 & 2 & 1 & - & $72 \%$ & $68 \%$ & 0 & 1 & 0 & $40 \%$ & $2 \%$ & $0 \%$ \\
\hline 21 & 308 & 5 & 0 & 2 & 2 & - & $85 \%$ & $70 \%$ & 0 & 1 & 0 & $56 \%$ & $4 \%$ & $0 \%$ \\
\hline 22 & 214 & 4 & 0 & 2 & 1 & - & $89 \%$ & $84 \%$ & 0 & 1 & 0 & $73 \%$ & $4 \%$ & $16 \%$ \\
\hline 23 & 214 & 4 & 0 & 2 & 1 & - & $85 \%$ & $94 \%$ & 0 & 1 & 0 & $92 \%$ & $5 \%$ & $64 \%$ \\
\hline 24 & 256 & 6 & 1 & 2 & 1 & $72 \%$ & $79 \%$ & $30 \%$ & 0 & 2 & 0 & $70 \%$ & $4 \%$ & $37 \%$ \\
\hline 25 & 214 & 5 & 0 & 2 & 1 & - & $66 \%$ & $84 \%$ & 0 & 2 & 0 & $77 \%$ & $3 \%$ & $44 \%$ \\
\hline Avg. & 224.16 & 4.80 & 0.60 & 2.00 & 0.84 & $80 \%$ & $74 \%$ & $77 \%$ & 0.00 & 1.36 & 0.00 & $76 \%$ & $3 \%$ & $31 \%$ \\
\hline
\end{tabular}

It is clear from the table that the medium-duty truck is the most preferred resource in the fleet, as it establishes an adequate balance between the energy-efficient but capacity-inefficient light-duty truck, and the energy-inefficient but capacity-efficient heavy-duty truck. Moreover, capacity usage in all cases for all vehicle types is very well distributed, for 
which some contribution could be attributed to multiple-trips possibility. It is also interesting to see that when a vehicle is assigned to an extra round of trip it is not under-utilised in terms of its capacity.

\subsection{The Pareto front of the SPRP}

All the test instances with 5 customers were solved to multi-objective optimality for the generation of the full set of the ND points on the true Pareto fronts using the approach discussed in section 5 of the paper, i.e. embedding the PEP-based MILP within the framework of the QSM (QSM+PEP-based MILP). Again, an alternative approach based on the integration of the QSM with the TDSP-based MILP (QSM+TDSP-based MILP) is considered to further see the effect of suboptimal network reduction techniques on the generation of the true Pareto fronts. The QSM+TDSP-based MILP uses the TDSPbased MILP described earlier as the core optimisation problem. That is, a multi-graph based on the full set of the TDSPS between required nodes is considered, and the MILP described in (16)-(28) is solved on this multi-graph rather than on the original PEP-based multi-graph. Like the case of the QSM+PEP-based MILP, in each iteration of the QSM+TDSP-based MILP, for returning a ND vector, the two (M)IPs discussed in section 5 must be solved. For all test instances we had to calibrate only $\epsilon_{2}$ as the first objective is integer, and hence considered $\epsilon_{1}=1$, and $\epsilon_{2}=0.01\left(\epsilon_{1}\right.$ and $\epsilon_{2}$ are QSM parameters). Also, as earlier mentioned no time limit was applied on the solver to allow the full set of the ND points to be found.

In Table 4, the total number of ND points and the objective-wise values of the extreme points on the Pareto fronts found by each of the two approaches are shown. In this table, the columns $\mathrm{Obj}_{1}, \mathrm{Obj}_{2}$, and $\mathrm{Obj}_{3}$ represent the global minimum value found for the first objective (vehicle cost in $£$ ), the second objective (fuel consumption in lit.) and the third objective (travel time in mins.), respectively. The important column here is the column with the heading 'I $\prec$ II' which indicates the number of ND points found by the QSM+TDSP-based MILP which are strictly dominated by the ND points on the QSM+PEP-based SPRP Pareto front.

Table 4

Extreme points on the Pareto fronts generated by each approach

\begin{tabular}{|c|c|c|c|c|c|c|c|c|c|c|c|}
\hline \multirow{3}{*}{$\begin{array}{l}\text { Instance } \\
\#\end{array}$} & \multicolumn{5}{|c|}{ I: QSM+PEP-based MILP } & \multicolumn{6}{|c|}{ II: QSM+TDSP-based MILP } \\
\hline & \multirow{2}{*}{$\begin{array}{l}\text { \# of } \\
\text { ND } \\
\text { points }\end{array}$} & \multicolumn{3}{|c|}{ Extreme points } & \multirow{2}{*}{$\begin{array}{l}\text { Runtime } \\
\text { (min) }\end{array}$} & \multirow{2}{*}{$\begin{array}{l}\text { \# of } \\
\text { ND } \\
\text { points }\end{array}$} & \multicolumn{3}{|c|}{ Extreme points } & \multirow{2}{*}{$\mathrm{I} \prec \mathrm{II}$} & \multirow{2}{*}{$\begin{array}{l}\text { Runtime } \\
\text { (min) }\end{array}$} \\
\hline & & $\mathrm{Obj}_{1}$ & $\mathrm{Obj}_{2}$ & Obj3 & & & Obj1 & $\mathrm{Obj}_{2}$ & $\mathrm{Obj}_{3}$ & & \\
\hline 1 & 40 & 94 & 43.66 & 228 & 127.24 & 28 & 94 & 49.54 & 228 & 22 & 21.78 \\
\hline 2 & 43 & 60 & 28.16 & 245 & 808.28 & 37 & 60 & 29.11 & 245 & 33 & 46.63 \\
\hline 3 & 50 & 94 & 38.86 & 222 & 150.20 & 12 & 94 & 41.41 & 222 & 10 & 10.13 \\
\hline 4 & 39 & 60 & 25.13 & 229 & 73.45 & 33 & 60 & 25.52 & 229 & 24 & 43.90 \\
\hline 5 & 31 & 94 & 36.96 & 267 & 166.40 & 12 & 94 & 38.46 & 267 & 10 & 12.73 \\
\hline 6 & 65 & 94 & 36.91 & 227 & 1095.91 & 41 & 94 & 38.35 & 227 & 34 & 175.08 \\
\hline 7 & 51 & 94 & 41.91 & 256 & 662.77 & 46 & 94 & 43.57 & 256 & 31 & 171.47 \\
\hline 8 & 79 & 94 & 41.52 & 270 & 222.03 & 72 & 94 & 42.11 & 270 & 70 & 123.31 \\
\hline 9 & 77 & 136 & 50.08 & 250 & 218.51 & 57 & 136 & 50.74 & 250 & 35 & 103.90 \\
\hline 10 & 101 & 154 & 67.75 & 323 & 616.62 & 78 & 154 & 68.46 & 323 & 43 & 299.35 \\
\hline 11 & 39 & 94 & 44.31 & 210 & 51.84 & 25 & 94 & 46.30 & 210 & 12 & 8.12 \\
\hline 12 & 41 & 94 & 25.97 & 192 & 657.65 & 28 & 94 & 26.89 & 192 & 19 & 116.95 \\
\hline 13 & 27 & 154 & 56.75 & 239 & 82.50 & 22 & 154 & 58.67 & 239 & 13 & 108.78 \\
\hline 14 & 82 & 136 & 41.90 & 234 & 133.68 & 55 & 136 & 43.31 & 234 & 43 & 67.65 \\
\hline 15 & 30 & 94 & 31.67 & 207 & 76.88 & 23 & 94 & 32.29 & 207 & 16 & 63.87 \\
\hline 16 & 100 & 94 & 41.04 & 247 & 982.92 & 88 & 94 & 41.18 & 247 & 82 & 775.78 \\
\hline 17 & 34 & 94 & 24.52 & 180 & 159.99 & 39 & 94 & 26.62 & 180 & 35 & 63.89 \\
\hline 18 & 53 & 60 & 33.98 & 231 & 177.97 & 13 & 60 & 34.81 & 231 & 6 & 8.22 \\
\hline 19 & 61 & 94 & 38.21 & 212 & 48.66 & 33 & 94 & 39.14 & 212 & 21 & 7.75 \\
\hline 20 & 41 & 94 & 25.97 & 192 & 566.37 & 28 & 94 & 26.89 & 192 & 19 & 111.30 \\
\hline 21 & 27 & 154 & 56.75 & 239 & 83.78 & 22 & 154 & 58.67 & 239 & 13 & 106.04 \\
\hline 22 & 82 & 136 & 41.90 & 234 & 108.65 & 55 & 136 & 43.31 & 234 & 43 & 70.34 \\
\hline
\end{tabular}




\begin{tabular}{lllllllllllll}
23 & 34 & 94 & 24.52 & 180 & 133.43 & 39 & 94 & 26.62 & 180 & 35 & 59.76 \\
24 & 53 & 60 & 33.98 & 231 & 129.54 & 13 & 60 & 34.81 & 231 & 6 & 7.87 & 7.29 \\
25 & 61 & 94 & 38.21 & 212 & 41.26 & 33 & 94 & 39.14 & 212 & 21 & 72 \\
\hline Average & 53.64 & 100.8 & 38.82 & 230.28 & 303.06 & 37.28 & 100.8 & 40.24 & 230.28 & 27.84 & 103.68 \\
\hline
\end{tabular}

It is observed that in several of the cases the number of the ND points found by the TDSP-based formulation is much less than what is found by the PEP-based one, and more importantly, many of these are strictly dominated. For example, there are $50 \mathrm{ND}$ points on the true Pareto front of instance \#3 found by the PEP-based formulation, and there are only 12 ND points on the Pareto front of the TDSP-based model; however, 10 of these are strictly dominated by the solutions on the true front, meaning that the TDSP-based approach has been in effect able to find only 2 ND points out of the 50 solutions. On average, the number of the ND points found by the TDSP-based formulation is 70\% of what is found by the PEP-based formulation, but around $75 \%$ of these are strictly dominated by the true front. Comparison of the extreme points, on the other hand, reveals that as expected the solutions with minimal vehicle cost and travel time could be found by the TDSP-based formulation; however, complying with the results in Table 2, in all cases a suboptimal solution for fuel consumption minimisation is yielded by the TDSP-based formulation.

It is worth mentioning that despite the size of the POSs (i.e. around 54 ND points on average) the full sets of ND points are generated within a very reasonable runtime of around 304 minutes on average, suggesting the successful integration of the QSM with the proposed MILP formulation.

Unlike bi-objective optimisation, visualisation of the Pareto front in the case of the multi-objective optimisation with more than two objectives is not easy. However, to provide the decision maker with a useful visual presentation of the tradeoffs among the ND points on the Pareto front, and aid her/him to select a solution that provides a suitable compromise among the objective values, we propose the use of enhanced heat maps similar to the one shown in Figure 6 . In this figure, the Pareto front of instance \#4 is selected and the percentage deviations from the absolute minimum in case of each objective function for all the 39 solutions on the considered front is shown. While it can be observed that there exists a significant trade-off among the three objectives of the SPRP and the minimisation of one objective can significantly deteriorate the value of the other two, with the help of the colour gradient, this figure makes it possible to visually locate the more balanced solutions.

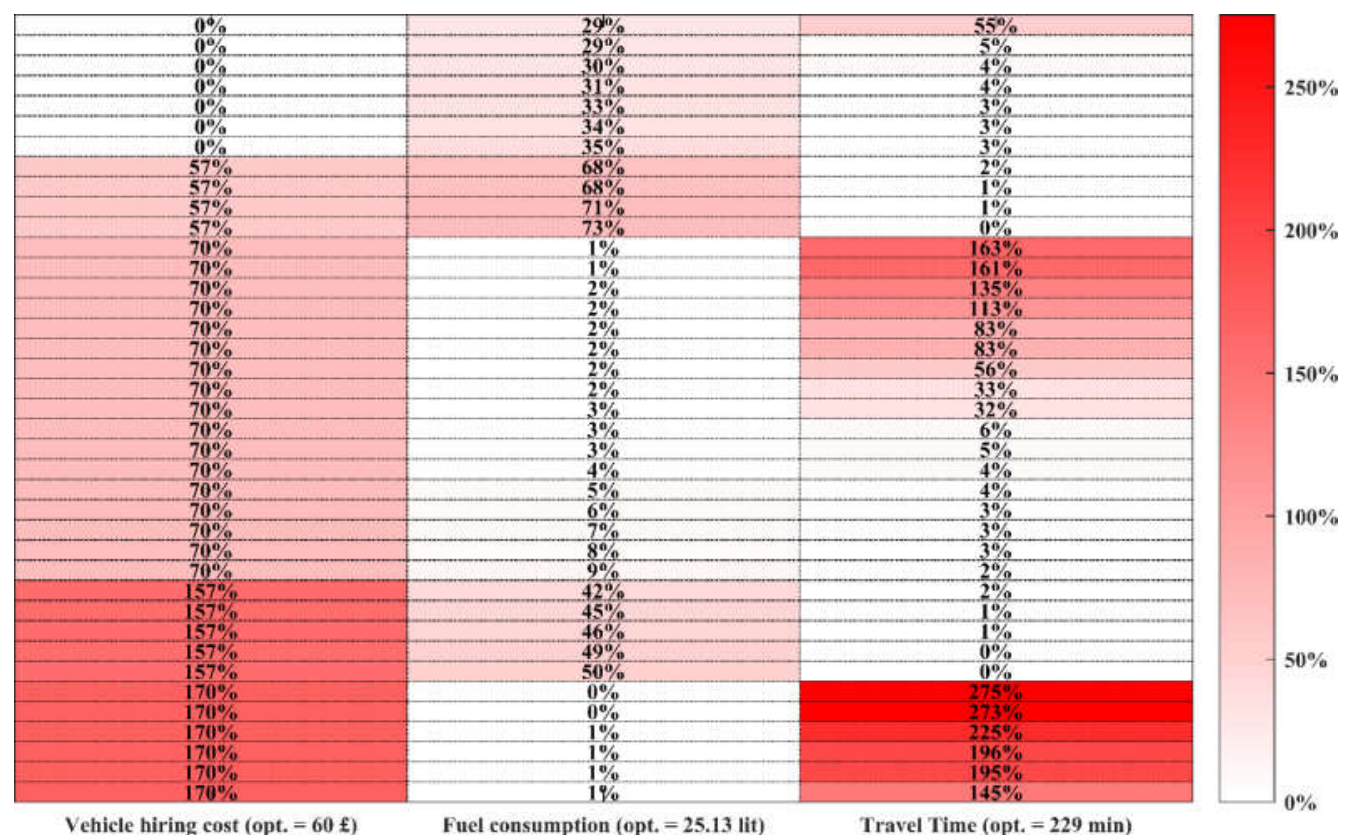

Figure 6 Heat map illustrating the ND points to a given SPRP instance 
It is also clear from Figure 6 that in the case of the considered instance the range of deviations in the travel time objective is much larger than the other two objectives, and it is particularly maximised when the solution tends to minimise fuel consumption. To investigate this further, we analyse that when one of the objectives is minimised, how much sacrifice is made in case of the other two objectives. As an average over all the instances with 5 customers, Figure 7 illustrates this trade-off. Similar to Figure 6, Figure 7 shows clearly that less sacrifice must be made in the other two objectives, when one minimises travel time, whereas the minimisation of fuel consumption can lead to a significant increase in the other two objectives.

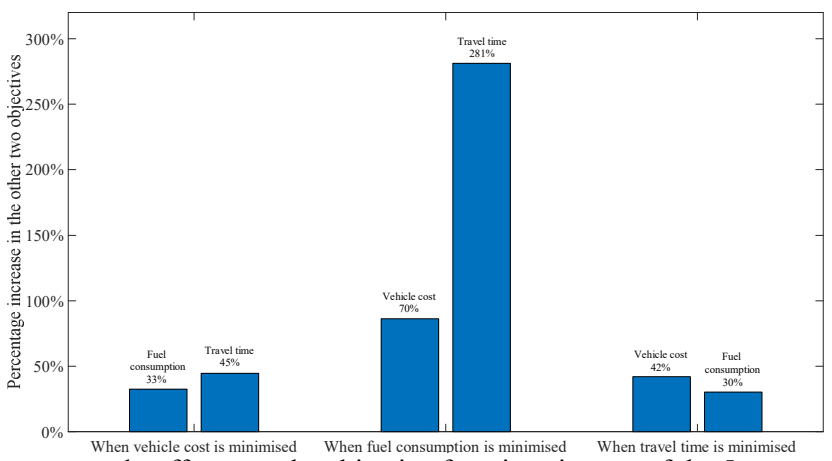

Figure 7 Average trade-off among the objective functions in case of the 5-customer instances

Finally, in Figure 8 for one instance we illustrate the routing patterns of the solution with minimum vehicle cost, the solution with minimum fuel consumption, and the solution with minimum travel time.

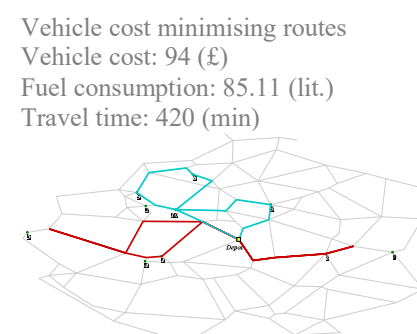

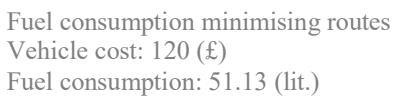

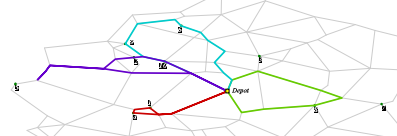

Travel time minimising routes

Vehicle cost: $94(£)$

Fuel consumption: 79.87 (lit)

Travel time: 398

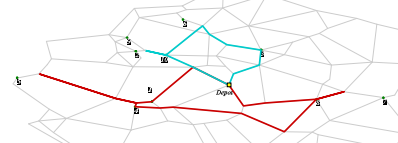

Figure 8 Routing patterns in case of the minimal objective value for each of the objectives

In this figure, in case of the minimum fuel consumption routes, two medium duty trucks are used for four routes (each making an extra round of trip), whereas in the other two cases all customers are served by one heavy-duty truck that also makes an extra round of delivery.

\subsection{Reliability of the constructed driving cycles}

In order to demonstrate the reliability of the proposed model for the generation of synthetic driving cycles, an extensive library of real-world driving cycles, consisting of over 19,000 different on-road driving cycles collected by Kancharla and Ramadurai (2018), are used as the benchmark set. For each cycle, the travel time and the distance of the cycle is fed into the proposed model, and the model is gotten to generate a synthetic cycle. Following the cycle generation, an empty lightduty truck (which is similar to the probe truck used in data collection in their study) is assumed to traverse both the real and the synthetic cycles, and the incurred fuel consumption based on the instantaneous CMEM in each case is computed. The percentage deviation of the incurred fuel consumption over the synthetic cycle from the incurred fuel consumption over the real driving cycle (i.e. $\left[\left(\right.\right.$ Fuel $_{\text {synthetic }}-$ Fuel $\left.\left.\left._{\text {real }}\right) / F u e l_{\text {real }}\right] \times 100\right)$ is then calculated. A descriptive statistics summary of the percentage deviations in all the 19,362 cases is presented in Table 5, and a histogram of these deviations is illustrated in Figure 9. 
Table 5

Descriptive statistics summary table of percentage deviation of the generated cycles from on road-cycles

\begin{tabular}{llll}
\hline Count & 19362 & Sample Variance & 0.000127964 \\
Mean & 0.018511164 & Kurtosis & 1.31344784 \\
Standard Error & $8.12959 \mathrm{E}-05$ & Skewness & 1.025999919 \\
Median & 0.016021384 & Range & 0.0983492 \\
Mode & 0.054775047 & Minimum & -0.020289727 \\
Standard Deviation & 0.011312111 & Maximum & 0.078059472 \\
\hline
\end{tabular}

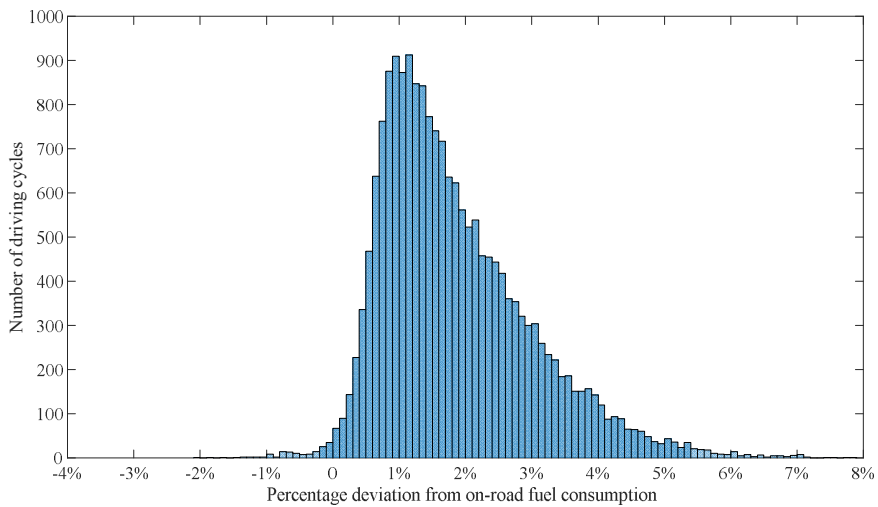

Figure 9 Histogram of percentage deviations of fuel consumption on synthetic cycles from on-road data

Based on Table 5, the proposed MILP can generate driving cycles, which have fuel consumption characteristics very close to real-life driving cycles, and on average lead to a fuel consumption estimation inaccuracy of less than $2 \%$. Based on the histogram in Figure 9, for over $75 \%$ of the cycles considered, deviation lies between 0 and $2.5 \%$.

Finally, it is useful to see the value of gaining this information and using truck A/D rates in fuel consumption estimation when routing on congested road networks. To this aim, we have solved all the instances with 10 customers to fuel consumption optimality when A/D rates are ignored and assumed zero all over the network, throughout the planning horizon. The performance of these solutions is then evaluated under the 'real' speed-time profiles; i.e. when the truck in practice accelerates and decelerates based on the generated driving cycles. With this, we are able to see to some extent the sub-optimality of these solutions and the estimation inaccuracy they contain. The results of these experiments are presented in Table 6.

Table 6

The effect of estimating fuel consumption inaccurately due to ignoring truck A/D rates

\begin{tabular}{lllll}
\hline Instance \# & Arcs & $\begin{array}{l}\text { Percentage } \\
\text { optimality gap }\end{array}$ & $\begin{array}{l}\text { Estimation } \\
\text { inaccuracy }\end{array}$ & $\begin{array}{l}\text { DCs generation } \\
\text { runtime (seconds) }\end{array}$ \\
\hline 1 & 129 & $11.67 \%$ & $75.51 \%$ & 294.90 \\
2 & 157 & $5.73 \%$ & $76.89 \%$ & 512.66 \\
3 & 160 & $2.80 \%$ & $61.08 \%$ & 320.29 \\
4 & 169 & $1.02 \%$ & $68.03 \%$ & 484.26 \\
5 & 185 & $2.06 \%$ & $56.64 \%$ & 351.54 \\
6 & 151 & $0.37 \%$ & $67.26 \%$ & 483.57 \\
7 & 191 & $2.13 \%$ & $57.33 \%$ & 317.61 \\
8 & 168 & $3.68 \%$ & $65.44 \%$ & 532.96 \\
9 & 120 & $9.59 \%$ & $81.36 \%$ & 1006.06 \\
10 & 172 & $2.97 \%$ & $66.49 \%$ & 469.92 \\
11 & 156 & $4.41 \%$ & $68.61 \%$ & 325.69 \\
12 & 130 & $4.23 \%$ & $71.33 \%$ & 514.18 \\
13 & 138 & $2.49 \%$ & $52.77 \%$ & 527.12 \\
14 & 190 & $4.74 \%$ & $73.33 \%$ & 459.93 \\
15 & 188 & $0.71 \%$ & $57.80 \%$ & 319.48 \\
16 & 142 & $3.04 \%$ & $58.43 \%$ & 463.71
\end{tabular}




\begin{tabular}{lllll}
17 & 155 & $0.84 \%$ & $58.82 \%$ & 617.72 \\
18 & 147 & $2.17 \%$ & $68.74 \%$ & 504.84 \\
19 & 188 & $3.53 \%$ & $45.77 \%$ & 673.53 \\
20 & 172 & $1.13 \%$ & $58.52 \%$ & 496.20 \\
21 & 188 & $2.90 \%$ & $75.05 \%$ & 480.11 \\
22 & 174 & $7.54 \%$ & $76.86 \%$ & 522.29 \\
23 & 150 & $2.96 \%$ & $65.74 \%$ & 450.84 \\
24 & 184 & $2.20 \%$ & $55.03 \%$ & 492.37 \\
25 & 145 & $8.57 \%$ & $79.10 \%$ & 531.54 \\
\hline
\end{tabular}

It must be noted that ignoring truck $\mathrm{A} / \mathrm{D}$ rates results in a completely different reduced network when the PEP is applied. As it is presented in Table 6, when A/D rates are ignored the PEP can only represent on average around $80 \%$ of all eligible road-paths that are identified in the case of the instantaneous CMEM with A/D data. As the table suggests, ignoring $\mathrm{A} / \mathrm{D}$ rates leads to suboptimal solutions in all cases.

In Table 6, we have also reported the total computational time required for the generation of all driving cycles for all road-links in the network at all time periods in case of each instance, under the column 'DCs generation runtime'. Observe that in comparison with field data collection, the required computational cost reported in the table can be considered insignificant.

\section{Discussion and concluding remarks}

In this paper, we introduced a realistic urban freight distribution model that can address traditional business and environmental objectives simultaneously while integrating several factors affecting fuel consumption, on the original roadway network. The proposed model is a variant of the well-known PRP, called the SPRP, and is a multi-objective, time and load dependent, fleet size and mix, emissions minimising vehicle routing and scheduling problem, with time windows, flexible departure times, and multi-trips on congested urban road networks. The paper focused mainly on a key complication arising from emissions minimisation in a time and load dependent setting, corresponding to the identification of the full set of the eligible road-paths between consecutive truck visits. It was shown that the state-of-the-art preprocessing approaches are unable to extract all such paths from the underlying roadway graph and thus lead to sub-optimal solutions with an optimality gap of as high as $12 \%$ in terms of fuel consumption. It was also observed that compared with the proposed approach in the paper, the other approach based on the TDSPs has a very limited ability to identify true ND points on the Pareto front in a multi-objective case, where it is only able to identify less than $18 \%$ of the true ND points on average. Further experiments on a real road network based on Chicago's arterial streets indicated that the set of eligible road-paths between a given origin/destination pair can be so large, and while TDSPs constitute a very limited subset of these paths, the PEP can identify them all.

All models in the paper are based on the instantaneous CMEM formula and can incorporate second-by-second speed variations and thus A/D rates for a more accurate estimation of fuel consumption. However, acknowledging the fact that such well-grained speed data are rarely available to the decision maker at the planning stage, the paper proposed a simple optimisation model for the construction of reliable spatiotemporal driving cycles that with very few model inputs and no parameter tuning can yield synthetic driving cycles that very well approximate the expected real-life fuel consumption of the truck. Experiments that are carried out on over 19000 different on-road driving cycles confirm an average overestimation of less than $2 \%$ for the proposed model. This model hence can replace the costly and time-consuming data acquisition phase for attaining reliable figures on the expected fuel consumption in routing applications.

The proposed model in the paper also shed light on an interesting opportunity to further cut down on GHG emissions and costs by using more energy-and-cost-efficient resources in the fleet multiple times during the planning horizon through multi-trip optimisation; especially in urban areas where trips are rather short, and trucks could be simply reloaded and 
dispatched for an extra round of trip. Multi-trip optimisation in a time-dependent setting, however, has never been studied before, and would constitute an interesting line of research due to new and previously unvisited challenges that it brings about when tackling real life size test instances.

The SPRP is a realistic and hence a very difficult problem to solve, and the main limitation of the current work lies in its inability in addressing large practical size SPRP instances. We are developing tailored multi-objective optimisation heuristics for the SPRP in a follow up paper to cope with this situation. While there are multiple research opportunities relevant to the study of EMVRPs directly on the roadway networks, we identify the incorporation of the effect of nonrecurrent congestion in the routing decisions through the development of real-time or stochastic variants of the SPRP as a significant line for future research. Moreover, as a recommendation for further research, the development of realistic problem instances that can reflect the real daily congestion patterns, and allow the analyses of different what-if scenarios for the departure time, depot location, customers' demands and time-windows negotiation, planning horizon alterations, and fleet size and mix decisions, can help gaining many practical insights for a logistics system operating in an urban area.

\section{Appendices}

\section{Appendix A: List of the notation and acronyms}

The following table presents the major notation used in the text. We have attempted to keep unique meaning for each notation to the greatest extent possible. In those cases where an item has additional uses, they should be clear from context. Only notations that are used in more than one section are presented, and additional notations that are used within specific sections are explained when used.

\begin{tabular}{|c|c|c|c|}
\hline Notation & Definition & $\begin{array}{l}\text { Notatio } \\
\mathrm{n}\end{array}$ & Definition \\
\hline$G$ & $\begin{array}{l}\text { A directed graph representing a real } \\
\text { roadway network }\end{array}$ & $h_{i j}$ & $\begin{array}{l}\text { Number of available speed observations during the planning } \\
\text { horizon for road-link }(i, j) \in A\end{array}$ \\
\hline$N$ & The set of network nodes in $G$ & $v_{t}$ & Observed speed at time instant $m_{t}$ \\
\hline$A$ & The set of directed road-links in $G$ & $a_{t}$ & Slope of the line segment connecting $v_{t}$ to $v_{t+1}$ \\
\hline$N_{0}$ & The set comprising the depot only & $Z_{i j}^{k \tau}$ & $\begin{array}{l}\text { UTM attribute of a road-link (or a road-path if it is explicitly } \\
\text { mentioned) for truck type } k \in K \text { at departure time } \tau\end{array}$ \\
\hline$N_{1}$ & The set of customer nodes & $\Gamma_{i j}^{\tau}$ & $\begin{array}{l}\text { RTM attribute of a road-link (or a road-path if it is explicitly } \\
\text { mentioned) at departure time } \tau\end{array}$ \\
\hline$N_{2}$ & The set of network junctions & $\mathcal{D}_{i j}$ & $\begin{array}{l}\text { The set of all minimum fuel consuming paths between } \\
\text { required nodes } i \text { and } j\end{array}$ \\
\hline$n$ & Number of customers & $\mathcal{E}_{i j}$ & $\begin{array}{l}\text { The set of all paths } p_{i j} \text { with non-dominated vectors } \\
{\left[t^{\tau}\left(p_{i j}\right), f_{f}^{k \tau}\left(p_{i j}\right)\right]}\end{array}$ \\
\hline$m$ & Number of network junctions & $A T T_{\mathrm{p}}$ & Tuple containing the attributes of path $\mathfrak{p}$ \\
\hline$K$ & The fleet of heterogeneous vehicles & $\mathcal{R}_{i j}^{k \tau}$ & $\begin{array}{l}\text { The set of retained eligible paths between nodes } i, j \in N_{R} \text { for } \\
\text { vehicle type } k \in K \text { at departure time } \tau\end{array}$ \\
\hline$\vartheta$ & The total number of vehicles in $K$ & $\mathcal{A T} \mathcal{T}_{i j}^{k \tau}$ & $\begin{array}{l}\text { The set of tuples containing the attributes of all paths retained } \\
\text { in } \mathcal{R}_{i j}^{k \tau}\end{array}$ \\
\hline $\mathbb{k}$ & The number of vehicle types & $\mathcal{M}_{i j}^{\tau}$ & The ordered set of the $k$ fastest paths at time instant $\tau$ \\
\hline$\mu_{k}$ & Curb weight of vehicle $k \in K$ & $r$ & $\begin{array}{l}\text { Maximum number trips a truck is allowed to make } \\
\text { during the planning horizon }\end{array}$ \\
\hline$Q_{k}$ & Maximum payload of vehicle $k \in K$ & $t_{i j p}^{t}$ & Travel time of road-path $(i, j, p) \in \hat{A}$ during time period $\mathbb{E}$ \\
\hline$c_{k}$ & Daily hiring fixed cost of vehicle $k \in K$ & $Z_{i j p}^{k \mathbb{k}}$ & UTM attribute of road-path $(i, j, p) \in \hat{A}$ during time period $\mathbb{E}$ \\
\hline$q_{i}$ & Demand requested by customer $i \in N_{1}$ & $\Gamma_{i j p}^{\mathbb{t}}$ & RTM attribute of road-path $(i, j, p) \in \hat{A}$ during time period $\mathbb{E}$ \\
\hline$w_{i}$ & Hard time window of customer $i \in N_{1}$ & $\mathcal{T}_{i j p}$ & $\begin{array}{l}\text { Time horizon dedicated to road-path }(i, j, p) \in \hat{A} \text { for } \\
\text { customised discretisation }\end{array}$ \\
\hline$e_{i}$ & Lower boundary of $w_{i}$ & $h_{i j p}$ & Number of time periods during $\mathcal{T}_{i j p}$ \\
\hline$l_{i}$ & Upper boundary of $w_{i}$ & $a_{i j p}^{t}$ & The lower boundary of time period $\mathbb{E} \in \mathcal{T}$ \\
\hline$s_{i}$ & Service time at customer $i \in N_{1}$ & $b_{i j p}^{t}$ & The upper boundary of time period $\mathbb{E} \in \mathcal{T}$ \\
\hline
\end{tabular}




\begin{tabular}{|c|c|c|c|}
\hline$T$ & The planning horizon & $x_{i j p}^{k \mathfrak{k}}$ & $\begin{array}{l}\text { Binary variable equal to } 1 \text { iff vehicle } k \in K \text { departs node } i \in \\
\hat{N} \text { during time period } \mathbb{E} \in \mathcal{T} \text { to go to node } j \in \hat{N} \text {, through } \\
\text { road-path }(i, j, p) \in \hat{A}\end{array}$ \\
\hline$d_{i j}$ & Distance of road-link $(i, j) \in A$ & $f_{i j p}^{k \Downarrow}$ & $\begin{array}{l}\text { Continuous variable to represents the size of load carried by } \\
\text { vehicle } k \in K \text { over the road-path }(i, j, p) \in \hat{A} \text { during time } \\
\text { period } \mathbb{E}\end{array}$ \\
\hline$t_{i j}^{\tau}$ & $\begin{array}{l}\text { The time-dependent travel time of road- } \\
\text { link (or a road-path if it is explicitly } \\
\text { mentioned) for departure time } \tau\end{array}$ & $y_{i j p}^{k \mathfrak{k}}$ & $\begin{array}{l}\text { Integer variable indicating the exact departure time from the } \\
\text { origin of path }(i, j, p) \in \hat{A} \text { given that it is departed by } \\
\text { vehicle } k \in K \text { during time period } \mathbb{E}\end{array}$ \\
\hline$N_{R}$ & The set of required nodes & $y_{\mathbb{N}}$ & The set of non-dominated points \\
\hline$p_{i j}$ & $\begin{array}{l}\text { A road-path that connects a pair of required } \\
\text { nodes } i, j \in N_{R} \text {. }\end{array}$ & $\mathcal{A}_{\max }$ & The maximum possible acceleration rate for a truck \\
\hline$p_{i j}$ & $\begin{array}{l}\text { The set of all paths between a pair of } \\
\text { required nodes } i, j \in N_{R}\end{array}$ & $\mathcal{D}_{\max }$ & The maximum possible deceleration rate for a truck \\
\hline$t^{\tau}\left(p_{i j}\right)$ & $\begin{array}{l}\text { The time-dependent travel time of road- } \\
\text { path connecting nodes } i \text { to } j \text { for departure } \\
\text { time } \tau\end{array}$ & $v_{U}$ & The maximum possible speed in the network \\
\hline$f_{f}^{k \tau}\left(p_{i j}\right)$ & $\begin{array}{l}\text { Fuel consumption of a truck } k \in K, \\
\text { carrying a load } f \in\left[0, Q_{k}\right] \text { at departure } \\
\text { time } \tau\end{array}$ & $v_{t}$ & Speed level at each second $t$ of a driving cycle \\
\hline$r$ & A route or a vehicle trip & $A C C_{t}$ & The acceleration rate during second $t-1$ until $t$ \\
\hline$r_{p}$ & A route-path & $D E C_{t}$ & The deceleration rate during second $t-1$ until $t$ \\
\hline$r_{p}^{\tau}$ & A route-trajectory & $x_{t}$ & $\begin{array}{l}\text { Binary decision variable equal to } 1 \text { iff vehicle accelerates } \\
\text { during second } t-1 \text { until } t\end{array}$ \\
\hline$t\left(r_{p}^{\tau}\right)$ & Travel time of a route trajectory $r_{p}^{\tau}$ & $\epsilon_{1}$ & QSM parameter \\
\hline$f^{k}\left(r_{p}^{\tau_{0}}\right)$ & $\begin{array}{l}\text { Fuel consumption of a truck } k \in K \text { over a } \\
\text { route-trajectory } r_{p}^{\tau}\end{array}$ & $\epsilon_{2}$ & QSM parameter \\
\hline
\end{tabular}

The following table presents the list of acronyms used within the paper:

\begin{tabular}{llll}
\hline Acronym & Meaning & Acronym & Meaning \\
\hline SPRP & Steiner Pollution Routing Problem & TDSP & Time-Dependent Shortest Path \\
PEP & Path Elimination Procedure & MOMILP & Multi-Objective MILP \\
A/D & Acceleration/Deceleration & POS & Pareto Optimal Set \\
UFD & Urban Freight Distribution & TDVRP & Time-Dependent VRP \\
$\mathrm{CO}_{2}$ & Carbon Dioxide & GPS & Global Positioning System \\
HGV & Heavy Goods Vehicle & UTM & Unrelated to Truck Mass \\
VRP & Vehicle Routing Problem & RTM & Related to the Truck Mass \\
GHG & Greenhouse Gas & MOCO & Multi-Objective Combinatorial Optimisation \\
EMVRP & Emissions Minimising Vehicle Routing & MOILP & Multi-Objective Integer Linear Programming \\
& Problem & & \\
PRP & Pollution Routing Problem & MOMILP & Multi-Objective Mixed Integer Linear Programming \\
TSP & Travelling Salesman Problem & MOLP & Multi-Objective Linear Programming \\
CMEM & Comprehensive Modal Emissions Model & MPT & Mathematical Programming Technique \\
ND & Non-Dominated & QSM & Quadrant Shrinking Method \\
MILP & Mixed Integer Linear Programming & ITS & Intelligent Transportation System \\
\hline
\end{tabular}

\section{Appendix B: Analysis of the PEP on Chicago's road network}

A publicly available real-world urban road network with time-dependent speed observations is used for further experimentation with the PEP. This is based on the Chicago's arterial (non-freeway) streets (https://data.cityofchicago.org) (Figure 10). We use the graph and the traffic updates provided by Dokka and Goerigk (2017) based on this database. They record 98 speed observations in 15-minute intervals over a time horizon of 24 hours spanning Monday March $27^{\text {th }}, 2017$ morning to Tuesday March $28^{\text {th }}, 2017$ morning, for a graph containing 538 nodes and 1308 arcs. 


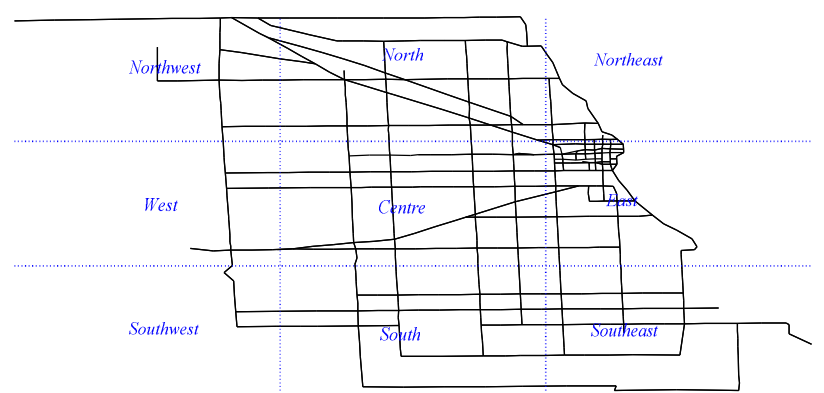

Figure 10 Chicago's arterial streets

A set of 11 source-sink pairs were selected on this road network, and a planning horizon of 12 hours from 07:00 to 19:00 was considered for experiments. To validate the performance of the PEP, an exhaustive approach for the exact identification of the full set of the eligible paths was used. This approach is based on the discretisation of the load range [0,26000] in $20 \mathrm{~kg}$ increments (1301 increments), and computation of the emissions minimising paths for all the resulting increments and feasible vehicle types, at all 720 possible departure times (every minute in the planning horizon). With this approach (called the Exact approach hereafter) we can identify the full set of all eligible paths and consider this set as the benchmark for the evaluation of the alternative approaches. In Table 7, the performance of the PEP and TDSP algorithms against the exact set of the eligible paths is reported. The reported runtime is based on the average of 10 runs.

Table 7

The performance of the PEP and TDSP algorithms against the Exact set of the eligible road-paths

\begin{tabular}{|c|c|c|c|c|c|c|c|}
\hline \multirow{2}{*}{ Pair } & \multirow{2}{*}{ Node Regions } & \multicolumn{3}{|c|}{ No. of Paths } & \multicolumn{3}{|c|}{ Runtime (seconds) } \\
\hline & & Exact & PEP & TDSP & Exact & PEP & TDSP \\
\hline$(499,481)$ & $(C, C)$ & 5 & 5 & 1 & 1400.97 & 4.21 & 0.87 \\
\hline$(7,314)$ & $(E, E)$ & 2 & 2 & 2 & 1110.91 & 3.96 & 0.90 \\
\hline$(106,325)$ & $(N, N)$ & 9 & 9 & 4 & 955.36 & 4.88 & 0.58 \\
\hline$(426,117)$ & $(S, S)$ & 6 & 6 & 2 & 984.09 & 3.21 & 0.46 \\
\hline$(3,72)$ & $(W, W)$ & 1 & 1 & 1 & 1330.73 & 3.71 & 0.80 \\
\hline$(20,175)$ & $(C, E)$ & 16 & 16 & 6 & 1505.43 & 3.60 & 0.89 \\
\hline$(19,325)$ & $(N W, N)$ & 10 & 10 & 4 & 963.59 & 2.49 & 0.90 \\
\hline$(49,111)$ & $(S, S E)$ & 9 & 9 & 5 & 2303.67 & 3.65 & 1.17 \\
\hline$(82,55)$ & $(N, S)$ & 18 & 18 & 7 & 1407.33 & 3.46 & 1.10 \\
\hline$(3,15)$ & $(W, E)$ & 28 & 28 & 14 & 1805.42 & 4.13 & 0.77 \\
\hline$(47,430)$ & $(S W, \mathrm{NE})$ & 46 & 46 & 24 & 1760.21 & 4.34 & 0.64 \\
\hline
\end{tabular}

According to Table 7, while the PEP is able to exactly identify all of the eligible paths in a very appealing runtime, the TDSP can identify only less than $50 \%$ of the eligible paths in most of the cases. Note that this also implies that adding the shortest distance path to the set of the TDSPs (if it is not already there) as proposed by Huang et al. (2017) cannot help much. This table also suggests that the number of the paths to retain is a pretty much relative value depending on several factors, and it is not possible to issue any prescription on a reliable number of paths to retain as in the k-shortest path network reduction technique of Androutsopoulos and Zografos (2017).

The percentage of time that the fuel consumption minimising path for a full truck of a given type, i.e. $p_{1}$, has been not the same as the fuel consumption minimisation path for the same truck type with no load, i.e. $p_{2}$, (see Proposition 1 ) in our experiments is also illustrated in the stacked bar chart in Figure 11 for all the 11 source-sink pairs in case of each vehicle type. This is the ratio of time instants out of the total 720 minutes in the time-horizon when the difference is observed. 


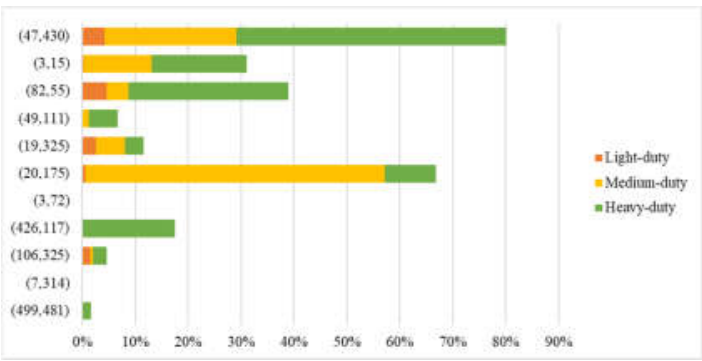

Figure 11 The percentage of time that $p_{1} \neq p_{2}$ for each truck type

Appendix C: The iterative algorithm for generating the network-wide driving cycles

Algorithm C.1 presents an iterative algorithm for the generation of all driving cycles for all road-links in the network at all time periods. To speed up this procedure, two things are done; firstly, we set the relative MIP gap tolerance of the CPLEX mixed integer programming setting to 0.01 , and the global time limit to 3 seconds. That is, if an optimal solution or a solution with 1\% MIP gap is observed in less than 3 seconds, it is accepted; otherwise, the solution that is returned by CPLEX after 3 seconds is accepted and used in the calculation of the road-link attributes (we never encountered a solution with over 5\% optimality gap with this setting). While CPLEX usually requires a few seconds to close the MIP gap and return the optimal solution, in almost all our observations, a solution with 1\% MIP gap is returned within fractions of a second. This solution is either the same as the optimal solution or very marginally different from that. Secondly, we store the obtained information iteratively in a hash table to use in later iterations. Indeed, the $D C_{i j}^{\mathfrak{t}}$ MILP relies mainly on two pieces of information; i.e. $d_{i j}$ and $t_{i j}^{\sharp}$. If we use $\left\lceil d_{i j}\right\rceil$ instead of $d_{i j}$, then the combination $\left\lceil d_{i j}\right\rceil$ and $t_{i j}^{\natural}$ is repeatedly observed for many road-links at different time periods. Note that the effect of this rounding up of distances on the ultimate values for the UTM and RTM attributes of the road-link is very negligible. Hence, in each iteration of the algorithm once the cycle is returned by the $D C_{i j}^{\mathbb{t}}$ MILP and the UTM and RTM attributes of the road link are computed, they are stored in the hash table $(H T)$ with their key $\left(\left[d_{i j}\right\rceil, t_{i j}^{\mathfrak{t}}\right)$. In following iterations before calling CPLEX to solve the $D C_{i j}^{\mathfrak{t}}$ MILP, the key is checked with the hash table to see if UTM and RTM values could be directly obtained from the table. Observe that at the end of the algorithm execution, it only needs to return the hash table, from which all UTM and RTM attributes of all road-links at all time periods could be extracted.

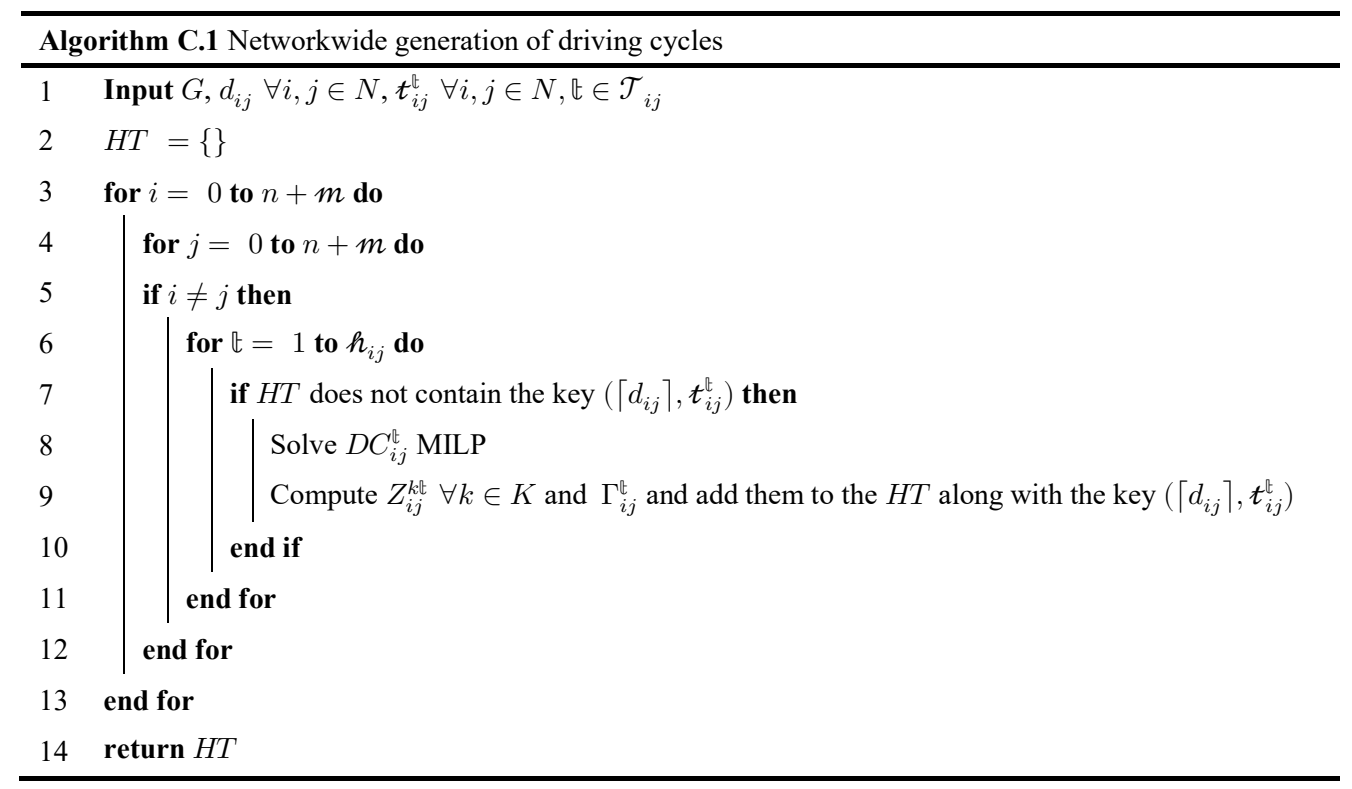




\section{References}

Allen, J., Browne, M., \& Piecyk, M. (2017). Assessing the European Commission's target of essentially CO2-free city logistics in urban centres by 2030. CityLab.

Alves, M. J., \& Clímaco, J. (2007). A review of interactive methods for multiobjective integer and mixed-integer programming. European Journal of Operational Research 180 (1), 99-115.

Androutsopoulos, K. N., \& Zografos, K. G. (2012). A bi-objective time-dependent vehicle routing and scheduling problem for hazardous materials distribution. EURO Journal on Transportation and Logistics 1 (1-2), 157-183.

Androutsopoulos, K. N., \& Zografos, K. G. (2017). An integrated modelling approach for the bicriterion vehicle routing and scheduling problem with environmental considerations. Transportation Research Part C 82, $180-209$.

Barth, M., Scora, G., \& Younglove, T. (2004). Modal emissions model for heavy-duty diesel vehicles. Transportation Research Record: Journal of the Transportation Research Board (1880), 10-20. 1250.

Bektas, T., \& Laporte, G. (2011). The Pollution-Routing Problem. Transportation Research Part B 45 (8), $1232-$

Bektaş, T., Ehmke, J. F., Psaraftis, H. N., \& Puchinger, J. (2019). The role of operational research in green freight transportation. European Journal of Operational Research 274(3), 807-823.

Belliss, G. (2004). Detailed Speed and Travel Time Surveys Using Low-Cost GPS Equipment. IPENZ. Wellington, New Zealand.

Ben Ticha, H., Absi, N., Feillet, D., \& Quilliot, A. (2018). Vehicle routing problems with road-network information: State of the art. Networks 72(3), 393-406.

Bokare, P. S., \& Maurya, A. K. (2017). Acceleration-deceleration behaviour of various vehicle types. Transportation research procedia $25,4733-4749$.

Boland, N., Charkhgard, H., \& Savelsbergh, M. (2016). The L-shape search method for triobjective integer programming. Mathematical Programming and Computation 8 (2), 217-251.

Boland, N., Charkhgard, H., \& Savelsbergh, M. (2017). The Quadrant Shrinking Method: A simple and efficient algorithm for solving tri-objective integer programs. European Journal of Operational Research 260 (3), $873-885$.

Byon, Y., Shalaby, A., \& Abdulhai, B. (2006). Travel time collection and traffic monitoring via GPS technologies. Proceedings of the 9th International IEEE Conference on Intelligent Transportation Systems (ITSC 2006) (pp. 677-682). Toronto, Canada: IEEE.

Çimen, M., \& Soysal, M. (2017). Time-dependent green vehicle routing problem with stochastic vehicle speeds: An approximate dynamic programming algorithm. Transportation Research Part D: Transport and Environment 54, 82-98.

Coello, C. A., Lamont, G. B., \& Van Veldhuizen, D. A. (2007). Evolutionary algorithms for solving multi-objective problems (Vol. 5). New York: Springer.

Cornuéjols, G., Fonlupt, J., \& Naddef, D. (1985). The traveling salesman problem on a graph and some related integer polyhedra. Mathematical programming 33 (1), 1-27.

Demir, E., Bektaş, T., \& Laporte, G. (2012). An adaptive large neighborhood search heuristic for the pollution-routing problem. European Journal of Operational Research 223 (2), 346-359.

Demir, E., Bektaş, T., \& Laporte, G. (2014). The bi-objective Pollution-Routing Problem. European Journal of Operational Research 232 (3), 464-478.

Dhaenens, M., Lemesre, J., \& Talbi, E. (2010). K-PPM: a new exact method to solve multi-objective combinatorial optimization problems. European Journal of Operational Research 200 (1), 45-53.

Dokka, T., \& Goerigk, M. (2017). An Experimental Comparison of Uncertainty Sets for Robust Shortest Path Problems. In G. D'Angelo, \& T. Dollevoet, 17th Workshop on Algorithmic Approaches for Transportation Modelling, Optimization, and Systems (ATMOS 2017), OpenAccess Series in Informatics (OASIcs), vol. 59 (pp. 16:1-16:13). Dagstuhl, Germany.

Ehmke, J. F., Campbell, A. M., \& Thomas, B. W. (2016). Vehicle routing to minimize time-dependent emissions in urban areas. European Journal of Operational Research 251 (2), 478-494.

Ehmke, J. F., Campbell, A. M., \& Thomas, B. W. (2018). Optimizing for total costs in vehicle routing in urban areas. Transportation Research Part E: Logistics and Transportation Review 116, 242-265.

European Comission. (2011). White Paper on Transport: Roadmap to a Single European Transport Area: Towards a Competitive and Resource-efficient Transport System. Publications Office of the European Union (Directorate-General for Mobility and Transport). 
Figliozzi, M. A. (2010). Vehicle routing problem for emissions minimization. Transportation Research Record: Journal of the Transportation Research Board 2197, 1-7.

Figliozzi, M. A. (2011). The impacts of congestion on time-definitive urban freight distribution networks CO2 emission levels: Results from a case study in Portland, Oregon. Transportation Research Part C 19 (5), $766-778$.

Franceschetti, A., Honhon, D., Woensel, T. V., Bektas, T., \& Laporte, G. (2013). The time-dependent pollutionrouting problem. Transportation Research Part B 56, 265-293.

Hamacher, H. W., Ruzika, S., \& Tjandra, S. A. (2006). Algorithms for time-dependent bicriteria shortest path problems. Discrete optimization 3 (3), 238-254.

Horn, M. E. (2000). Efficient modeling of travel in networks with time-varying link speeds. Networks 36 (2), 80-90.

Huang, Y., Zhao, L., Van Woensel, T., \& Gross, J. P. (2017). Time-dependent vehicle routing problem with path flexibility. Transportation Research Part B 95, 169-195.

Ichoua, S., Gendreau, M., \& Potvin, J.-Y. (2003). Vehicle dispatching with time-dependent travel times. European Journal of Operational Research 144 (2), 379-396.

Kancharla, S., \& Ramadurai, G. (2018). Incorporating driving cycle based fuel consumption estimation in green vehicle routing problems. Sustainable Cities and Society (40), 214-221.

Kara, I., Kara, B. Y., \& Yetis, M. K. (2007). Energy minimizing vehicle routing problem. International Conference on Combinatorial Optimization and Applications (pp. 62-71). Berlin, Heidelberg: Springer.

Koç, Ç., Bektaş, T., Jabali, O., \& Laporte, G. (2014). The fleet size and mix pollution-routing problem. Transportation Research Part B 70, 239-254.

Kramer, R., Maculan, N., Subramanian, A., \& Vidal, T. (2015). A speed and departure time optimization algorithm for the pollution-routing problem. European Journal of Operational Research 247 (3), 782-787.

Lee, R. J., Sener, I. N., \& Mullins III, J. A. (2016). An evaluation of emerging data collection technologies for travel demand modeling: from research to practice. Transportation Letters 8 (4), 181-193.

Letchford, A. N., Nasiri, S. D., \& Theis, D. O. (2013). Compact formulations of the Steiner Traveling Salesman Problem and related problems. European Journal of Operational Research 228 (1), 83-92.

Lokman, B., \& Köksalan, M. (2013). Finding all nondominated points of multi-objective integer programs. Journal of Global Optimisation 57 (2), 347-365.

MDS Transmodal. (2012). DG MOVE European Commission: Study on urban freight transport. Final report. Chester, UK: MDS Transmodal Limited.

Olivera, A., \& Viera, O. (2007). Adaptive memory programming for the vehicle routing problem with multiple trips. Computers \& Operations Research 34 (1), 28-47.

Orda, A., \& Rom, R. (1990). Shortest-path and minimum-delay algorithms in networks with time-dependent edgelength. Journal of the Association for Computing Machinery 37 (3), 607-625.

Özlen, M., \& Azizoglu, M. (2009). Multi-objective integer programming: a general approach for generating all nondominated solutions. European Journal of Operational Research 199 (1), 25-35.

Özlen, M., Burton, B. A., \& MacRae, C. A. (2014). Multi-objective integer programming: an improved recursive algorithm. Journal of optimisation theory and applications 160 (2), 470-482.

Qian, J., \& Eglese, R. (2016). Fuel emissions optimization in vehicle routing problems with time-varying speeds. European Journal of Operational Research 248 (3), 840-848.

Silvas, E., Hereijgers, K., Peng, H., Hofman, T., \& Steinbuch, M. (2016). Synthesis of realistic driving cycles with high accuracy and computational speed, including slope information. IEEE Transactions on Vehicular Technology 65 (6), 4118-4128.

Sylva, J., \& Crema, A. (2004). A method for finding the set of non-dominated vectors for multiple objective integer linear programs. European Journal of Operational Research 158 (1), 46-55.

Turkensteen, M. (2017). The accuracy of carbon emission and fuel consumption computations in green vehicle routing. European Journal of Operational Research 262 (2), 647-659.

Ubeda, S., Arcelus, F. J., \& Faulin, J. (2011). Green logistics at Eroski: A case study. International Journal of Production Economics 131 (1), 44 -51.

Wang, M., Daamen, W., Hoogendoorn, S., \& Van Arem, B. (2011). Estimating acceleration, fuel consumption, and emissions from macroscopic traffic flow data. Transportation Research Record: Journal of the Transportation Research Board 2260, 123-132. 
Xiao, Y., \& Konak, A. (2016). The heterogeneous green vehicle routing and scheduling problem with time-varying traffic congestion. Transportation Research Part E 88, 146-166.

Xiao, Y., Zhao, Q., Kaku, I., \& Xu, Y. (2012). Development of a fuel consumption optimization model for the capacitated vehicle routing problem. Computers \& Operations Research 39 (7), 1419-1431.

Zegeye, S. K., De Schutter, B., Hellendoorn, J., Breunesse, E. A., \& Hegyi, A. (2013). Integrated macroscopic traffic flow, emission, and fuel consumption model for control purposes. Transportation Research Part C 31, $158-171$.

Ziliaskopoulos, A. K., \& Mahmassani, H. S. (1993). Time-dependent, shortest-path algorithm for real-time intelligent vehicle highway system applications. Transportation Research Record: Journal of the Transportation Research Board 1408, 94-100. 